Revue musicale OICRM

\title{
Bilitis après Debussy. Hommage, influence, prise de distance ?
}

\section{Federico Lazzaro}

Volume 2, numéro 1, 2014

La réception de Debussy au XXe siècle. Incidences, influences et autorité

URI : https://id.erudit.org/iderudit/1055851ar

DOI : https://doi.org/10.7202/1055851ar

Aller au sommaire du numéro

Éditeur(s)

OICRM

ISSN

2368-7061 (numérique)

Découvrir la revue

Citer cet article

Lazzaro, F. (2014). Bilitis après Debussy. Hommage, influence, prise de distance

? Revue musicale OICRM, 2(1), 159-190. https://doi.org/10.7202/1055851ar

\section{Résumé de l'article}

Composer des mélodies issues des Chansons de Bilitis de Pierre Louÿs dans la France de la première moitié du XXe siècle impliquait évidemment une confrontation directe avec le recueil de Debussy, paru en 1899. Les comptes rendus des concerts à Paris dans les années 1920-1930, alors que le processus de canonisation de Debussy battait son plein, nous permettent d'étudier le discours autour des compositeurs proposant leurs propres Chansons de Bilitis. Le cycle du maître - très joué lui-même - y est souvent cité comme œuvre de référence, et devait forcément être pris en compte par les compositeurs se confrontant aux Chansons. Ainsi, en étudiant les nouvelles Bilitis, il est pertinent de s'interroger sur les attitudes des compositeurs envers l'oeuvre debussyste : est-ce que les nouvelles Bilitis se veulent comme un hommage explicite à Debussy ? Sont-elles plutôt indirectement influencées par le recueil du maître ? Ou bien prennent-elles une distance nette de leur illustre prédécesseur ? Deux auteurs en particulier font l'objet de cette étude comparée : Charles Koechlin et Georges Dandelot. Leur choix de textes parmi les Chansons de Louÿs ainsi que la dramaturgie et les techniques compositionnelles employées sont analysées ici. Les mélodies de Koechlin se révèlent très éloignées de l'imaginaire grec de Debussy, tandis que Dandelot bascule entre l'imitation du style debussyste et l'appropriation décontextualisée de certains de ses traits distinctifs. La comparaison du cycle debussyste avec les « autres " Chansons de Bilitis donne des indices sur la réception compositionnelle de Debussy et n'est pas sans faire ressortir les spécificités stylistiques et esthétiques du recueil debussyste.
Ce document est protégé par la loi sur le droit d'auteur. L'utilisation des services d'Érudit (y compris la reproduction) est assujettie à sa politique d'utilisation que vous pouvez consulter en ligne.

https://apropos.erudit.org/fr/usagers/politique-dutilisation/ 


\title{
Bilitis après Debussy. Hommage, influence, prise de distance?
}

\author{
Federico Lazzaro
}

\section{Résumé}

Composer des mélodies issues des Chansons de Bilitis de Pierre Louÿs dans la France de la première moitié du $\mathrm{xx}^{\mathrm{e}}$ siècle impliquait évidemment une confrontation directe avec le recueil de Debussy, paru en 1899. Les comptes rendus des concerts à Paris dans les années 1920-1930, alors que le processus de canonisation de Debussy battait son plein, nous permettent d'étudier le discours autour des compositeurs proposant leurs propres Chansons de Bilitis. Le cycle du maître - très joué lui-même - y est souvent cité comme œuvre de référence, et devait forcément être pris en compte par les compositeurs se confrontant aux Chansons. Ainsi, en étudiant les nouvelles Bilitis, il est pertinent de s'interroger sur les attitudes des compositeurs envers l'œuvre debussyste : est-ce que les nouvelles Bilitis se veulent comme un hommage explicite à Debussy ? Sontelles plutôt indirectement influencées par le recueil du maître ? Ou bien prennentelles une distance nette de leur illustre prédécesseur ? Deux auteurs en particulier font l'objet de cette étude comparée : Charles Koechlin et Georges Dandelot. Leur choix de textes parmi les Chansons de Louÿs ainsi que la dramaturgie et les techniques compositionnelles employées sont analysées ici. Les mélodies de Koechlin se révèlent très éloignées de l'imaginaire grec de Debussy, tandis que Dandelot bascule entre l'imitation du style debussyste et l'appropriation décontextualisée de certains de ses traits distinctifs. La comparaison du cycle debussyste avec les " autres " Chansons de Bilitis donne des indices sur la réception compositionnelle de Debussy et n'est pas sans faire ressortir les spécificités stylistiques et esthétiques du recueil debussyste.

Mots clés : Chansons de Bilitis ; Debussy ; influence ; mélodie française.

\begin{abstract}
The composition of Chansons de Bilitis by French composers in the first decades of 20th century implied a direct confrontation with Debussy's cycle, published in 1899. Concert reviews of the 1920s-1930s-when the canonization of Debussy was at its high - offer interesting material for studying the discourse about the composers who created their own Chansons de Bilitis. Reviews often cite Debussy's cycle-which was performed constantly - as a reference. Composers could not write their own Bilitis without considering Debussy's. Therefore, the study of the new Chansons brings up relevant questions about the composers' attitude towards Debussy's work: Are more recent Bilitis an explicit homage to Debussy? Are they influenced by the master's work in a more implicit way? Or do they definitely take a distance from their illustrious predecessor? Two authors in particular are the object of the present comparative
\end{abstract}


study: Charles Koechlin and Georges Dandelot. This analysis focuses on their text choice, dramaturgy and compositional technique. 's mélodies are very far from Debussy's imagined Greece, while Dandelot toggles between the imitation of Debussy style and the decontextualized appropriation of some of its distinctive features. The comparison between the Chansons de Bilitis by Debussy and by other composers is relevant about the compositional reception of Debussy. Moreover, some stylistic and aesthetic specificities of Debussy's cycle emerge from the comparative analysis.

Keywords: Chansons de Bilitis; Debussy; influence; French mélodie.

Mesdemoiselles Bilythis

Vous allez (et qu'un ample bis

Heurtant vos blanches mains de lys

Les change en roses mains de rose)

Voir, après avoir vu gratis Des Grecs de mille neuf cent dix,

Que cela se passait jadis

Exactement la même chose.

- Cocteau ([1910]1981, p. 1422).

Debussy a été le premier à composer et à publier des mélodies puisant dans les Chansons de Bilitis de Pierre Louÿs, mais il ne fut pas le seul. Composer sur ces textes après Debussy dans la France de la première moitié du xx siècle comportait évidemment une confrontation directe avec le recueil du maître, paru en 1899 : hommage, influence, prise de distance ? Nous chercherons à le comprendre au cours des pages suivantes, où des mélodies de Charles Koechlin et Georges Dandelot seront analysées par rapport à celles de Debussy, à travers une comparaison du choix des textes, de la dramaturgie musicale et des matériaux musicaux utilisés ${ }^{1}$. Le choix de nous limiter ici à l'analyse des Bilitis de Koechlin et Dandelot - parmi les dizaines de mélodies sur les textes de Louÿs composées en France dans les mêmes années - est dicté par la particularité du cas de Koechlin (son hommage à la Bilitis debussyste se trouve dans un autre recueil que ses Chansons de Bilitis) ainsi qu'à l'ampleur du recueil de Dandelot (quinze chansons, tandis qu'en général les autres compositeurs se limitent à deux ou trois).

Les comptes rendus des concerts à Paris dans les années 1920-1930, alors que le processus de canonisation de Debussy battait son plein, nous permettent d'étudier le discours autour des compositeurs proposant leurs propres Chansons de Bilitis. Parallèlement, l'analyse comparée du cycle debussyste avec les " autres " Chansons de Bilitis donne des indices sur la réception compositionnelle de Debussy et n'est pas sans faire ressortir les spécificités stylistiques et esthétiques du recueil debussyste.

$1 \quad$ Flothuis a proposé un premier aperçu des rapports entre les Chansons de Bilitis de Debussy, Koechlin et Dandelot. Il affirme que «Debussy a créé - comme souvent - un véritable modèle qui oblige tout autre compositeur à réfléchir en profondeur, avant de s'attaquer à la composition de textes de P. Louÿs. On n'écrit pas non plus impunément de nouvelle composition sur le texte de Goethe Gretchen am Spinnrade » (Flothuis 1996, p. 60). 
L'an zéro de l'histoire musicale des Chansons de Bilitis fut 1897, quand «La chevelure» de Debussy parut dans la revue L'image ; en 1899, l'éditeur Fromont publia le triptyque complet ("La flûte de Pan », "La chevelure », "Le tombeau des Naïades »). La première édition des Chansons de Louÿs date de 1895 (L'art indépendant) et la deuxième, revue et augmentée, de 1898 (Mercure de France) ${ }^{2}$.

En cette même année ("La chevelure » debussyste était déjà parue, pas encore les deux autres mélodies ${ }^{3}$ ), on entendit Bilitis, un « poème en 12 chants extrait des Chansons de Bilitis de Pierre Louÿs " par Rita Strohl, compositrice fascinée par le symbolisme. Le recueil paraîtra en $1900 \mathrm{chez}$ Toledo, enrichi par des illustrations de Notor « d'après de documents authentiques des musées d'Europe » (Strohl 1900) 4 .

Toujours en 1898, Charles Koechlin commença à composer ses Cinq Chansons de Bilitis, qu'il terminera en 1908 et ne publiera qu'en 1923 chez Sénart ${ }^{5}$. Entretemps, d'autres compositions inspirées du livre de Louÿs virent la lumière : un des Deux poèmes antiques (1899, publiés en 1908) de P. de Wailly ; quatre Chansons de Bilitis de P. Langlois (1904-05), trois de M. Pollet (1908), une de L. Robin (1920) ; l'organiste Marcel Dupré composa, lui aussi, Deux Chansons de Bilitis (parmi les Quatre Mélodies, op. 6, 1913) «nettement d'influence debussyste » (Delestre 1952, p. 40). La même année de la publication du recueil de Koechlin (1923), Georges Dandelot entama la composition de ses Quinze Chansons de Bilitis, qui sortiront en trois recueils chez Eschig entre 1929 et $1933^{6}$. En 1937, André-Marie Cuvelier publia une autre

2 Pour l'histoire du texte des Chansons de Bilitis, voir l'édition critique par Jean-Paul Goujon (Louÿs 1990), utilisée comme référence dans cette étude.

3 "La chevelure » fut publiée comme mélodie avant de l'être comme prose lyrique : absente de la première édition des Chansons de Bilitis, Louÿs en donna le manuscrit à Debussy qui lui avait demandé la permission de composer une pièce sur le texte d'une des Chansons (il proposait "La flûte de Pan ») ; voir Grayson 2001, p. 120-121.

4 Selon Le Coz (1991, cité par Launay 2006, p. 82), Rita Strohl devrait à son mari Émile l'idée de mettre en musique les Chansons de Bilitis. Le Coz affirme aussi que la création de cette œuvre aurait eu lieu à Bruxelles (Théâtre de la Monnaie) par Jane Bathori ; selon d'autres documents des archives privées de la compositrice, publiés dans un blog anonyme en 2009, la première aurait eu lieu à Paris (salle de la Bodinière), par « Mlle Menjaud accompagnée par l'auteur».

5 Quatre des cinq Chansons composées par Koechlin étaient déjà dans la première édition de 1895, sauf " Hymne à la nuit » que Louÿs ajouta pour l'édition de 1898 : il est donc possible que Koechlin commença à travailler sur ces textes lors de la parution de la deuxième édition (bien qu'il ne composa " Hymne à la nuit » qu'en 1908). La première audition de l'« Hymne à Astarté ", composée en 1898, eut lieu au Théâtre de la Bodinière le 14 mars 1899, donc un an avant la première des Chansons de Bilitis de Debussy (17 mars 1900, Société nationale, Salle Pleyel). Pour la datation des compositions de Koechlin, voir Orledge 1989.

6 Si la version éditée est pour chant et piano, les manuscrits conservés à F-Pn montrent que l'idée originaire de Dandelot était d'écrire un cycle de six chansons pour chant, quintette à cordes et piano. Le Ms 20010, daté " octobre 1923-février 1925 », contient trois mélodies pour les mêmes instruments. Les Ms 20011 et 20016 (ca 1925) présentent respectivement " La nuit » et " Les contes » pour chant et orchestre. En 1935, Le Monde musical nous informe que, pendant ses vacances estivales, Dandelot " a orchestré quelques-unes de ses Chansons de Bilitis pour Mlle Madeleine Grey qui les chantera chez Pasdeloup » (Anonyme 1935, p. 273). Ce concert eut lieu en février 1936 : " De ces quinze mélodies, 
sélection de 15 Chansons, à laquelle il fit suivre ultérieurement trois recueils, pour un total de 27 mélodies!

Entre la composition et la diffusion des mélodies de Dandelot, la deuxième moitié des années 1920 fut riche en Bilitis : il suffira de citer que dans le numéro du 15 juin 1925 du Courrier musical on trouve deux comptes rendus de concerts avec les Chansons de Debussy (qui seront aussi orchestrées par Maurice Delage en 1926). Parmi les nouvelles Chansons de Bilitis, Paris en entendit trois de Maurice Imbert ${ }^{7}$, quatre (instrumentales) de Dequin ${ }^{8}$, deux d'André Polonnais ${ }^{9}$. Même 1'humoriste Betove (Michel-Maurice Lévy) proposa des Chansons de Bilitis pour illustrer le pendant sérieux de sa double personnalité :

L'idée était intéressante de présenter en une même soirée Betove et son double Michel-Maurice Lévy, de montrer le fantaisiste à côté du musicien sérieux. [Lévy nous a délectés] aux inflexions sonores de Mme Croiza, dans des Chansons de Bilitis, dont elle cisela toutes les finesses. Puis, Betove joua du Wagner. On sait comment il le joue (Carol-Bérard 1925)...

Dans les années 1930, Bilitis s'impose dans le paysage musical parisien. En 1933, on assiste à l'exécution de deux recueils composés par deux femmes : Odette Fayau (Deux Chansons de Bilitis, "La lune aux yeux bleus " et "La pluie " ${ }^{10}$ ) et Jeanne Bernard (Trois Chansons de Bilitis ${ }^{11}$ ). En 1934, un autre triptyque fut publié (R. Thiel). Les jeunes étrangers s'intéressent aussi au sujet, comme c'est le cas de Gustavo Morales (Deux Chansons de Bilitis, 1930) et Roman Maciejewski (Trois Chansons de Bilitis ${ }^{12}$ ). Après la guerre, Rouart-Lerolle publiera Cinq Chansons de Bilitis de Robert Le Grand (1946) et Joseph Kosma écrira le produit le plus éloigné imaginable de l'esprit symboliste incarné par les poèmes de Louÿs et leur version debussyste : une opérette (Les chansons de Bilitis, livret de Jean Valmy et Marc-Cab), représentée au Théâtre des Capucines en 1953 et reprise au Petit Théâtre de Paris en 1960 sous le titre de Bilitis et l'Amour.

Le cas de Kosma s'inscrit dans une tradition parallèle, faite de clins d'œil plutôt que de filiation explicite, entre Bilitis, Debussy et d'autres compositeurs. En 1902, alors que les poèmes de Louÿs étaient encore fraîchement sortis de l'imprimerie et que les mélodies de Debussy n'étaient pas encore un classique, Émile Trepand composa une

ciselées avec amour, comme des bijoux alexandrins, d'une orchestration chatoyante et d'une diction fort spirituelle, P. Coppola avait extrait la scène de la Dispute ["Scène »] entre Bilitis et Mnasidika, la Berceuse de Bilitis et l'ironique Dialogue ["Conversation»] entre Bilitis et un passant : nous reprocherons peut-être à l'orchestre d'avoir un peu écrasé cette dernière, dans son accompagnement trop généreux » (Delage 1936).

7 Amis des Lettres français (voir Herstal 1925).

8 Société nationale, 16 mai 1925 (voir Himonet 1925 ; Duchesneau 1997, p. 286). Ce n'est pas le seul exemple de traduction instrumentale de Bilitis (voir ci-dessous).

9 Concert de Mme Destombes-Vallières, mars 1927 (voir Gibaudan 1927).

10 Société nationale, 11 février 1933 (voir Duchesneau 1997, p. 295).

11 Cercle musical de Paris, 3 mai 1933 (voir Curzon 1932).

12 Concert de l'Association des jeunes musiciens polonais, mars 1935 (voir Mangeot 1935). Demarquez (1935b) les décrit comme «tempétueuses » et s'exclame : «Dieu ! que le polonais leur va mal ! ». 
Bilitis! sur des paroles d'Arthur Bernède. Un autre poète, Max Rabusson, s'intéressa au sujet, inspirant deux Chansons de Bilitis au compositeur H. von Lohné (1908). Koechlin, pour sa part, composa entre 1902 et 1909 dix mélodies sur des textes à sujet grec d'Albert Samain ; il n'y a pas de Bilitis ici, mais dans la «nouvelle édition revue et corrigée par l'auteur » de ses mélodies, parue chez Philippo (sans date), Koechlin les a réunies dans un volume dédié "à la mémoire de Claude Debussy " : est-ce fortuit ${ }^{13}$ ?

Dans cette étude, nous nous concentrerons sur les mélodies de Koechlin (Bilitis et Samain) et sur le vaste cycle des Chansons de Bilitis de Dandelot - qui a eu une fortune assez remarquable à l'époque -, tout en en enrichissant l'analyse par l'entremise de liens aux autres recueils.

La comparaison des nouvelles mélodies issues des Chansons avec le cycle du maître n'est pas une constante dans la critique. Les comptes rendus des exécutions des pièces de Dandelot au début des années 1930 citent parfois Debussy comme un précédent encombrant :

Certes, dans la série des Chansons de Bilitis [...] il était difficile à M. G. Dandelot de ne pas éveiller parfois chez nous le souvenir précis et redoutable de trois pages saisissantes où le génie subtil, fantasque et profond de Claude Debussy s'est exprimé tout entier. Mais il a su y manifester une sincérité, une fraîcheur et une justesse d'accent plus rares que vous ne pensez par le temps qui court et qui ont paru avoir l'action la plus directe sur l'auditoire (Samazeuil 1932) ${ }^{14}$.

Selon d'autres critiques, au contraire, l'influence debussyste serait ce qui donne la valeur à la mélodie :

[...] trois Chansons de Bilitis de G. Dandelot, "Scène ", " Berceuse ", "Conversation ", données en première audition. La première se ressent violemment de l'influence debussyste... et c'est la meilleure des trois (Bertrand 1936) ${ }^{15}$.

13 Il s'agit du quatrième recueil des mélodies, qui contient dix pièces sur des textes de Samain et "Le vaisseau » sur un texte d'Edmond Haraucourt. Koechlin avait composé deux recueils sur des poèmes de Samain : Six mélodies, op. 31 et Quatre mélodies, op. 35 ; pour l'édition, il choisit neuf de ces mélodies et "Accompagnement " (qui faisait partie du même recueil que « Le vaisseau » de Haraucourt), à savoir les Quatre Mélodies, op. 28 (pour la mélodie substituée, voir la note 23). Les autres recueils de l'édition revue et corrigée sont dédiés "à la mémoire de ma mère ", "à ma femme ", "à ma nièce Guito ". Sur le rapport entre Koechlin et la Grèce antique, voir Corbier 2010 et ci-dessous.

14 Demarquez (1932) exprime une position similaire : "Parmi les Chansons de Bilitis, les meilleures furent, à mon avis, les six dernières, les autres évoquant un peu trop, par tant de neuvièmes et d'accords altérés, l'ombre de Debussy ». Selon Imbert (1929) (lui-même auteur de Trois chansons de Bilitis quelques années auparavant), Dandelot sort victorieux de la comparaison. À propos des Chansons de Bilitis d'Odette Fayau, Belvianes (1933) écrit que « malheureusement, malgré soi, l'on est amené à comparer, et... Debussy aussi a mis en musique des chansons de Bilitis ». Sur le processus de canonisation de Debussy à partir de sa mort, contrebalancé dans les mêmes années par un mouvement de démystification du maître, voir les communications présentées au colloque L'héritage de Claude Debussy (Montréal, 2012) lors de la séance "Canoniser Debussy » (Kelly 2012, Wheeldon 2012, Trottier 2012), ainsi que Trottier 2014.

15 Dans les deux pages qu'il consacre à Dandelot, Landormy (1943) ne fait cependant aucune référence à Debussy : "Georges Dandelot a une écriture très claire, où se sent la volonté de s'exprimer nettement. 
On aura remarqué que ce dernier compte rendu parle de trois mélodies ; en plusieurs autres occasions (Société nationale, 3 mai 1930 ; Société des concerts du Conservatoire, mars 1933 ; récital de Germaine Cernay, mai 1933 ; récital de Marcelle Gavarnier, janvier 1934), on compta au nombre de trois les mélodies de Dandelot au programme, exactement comme dans le cycle debussyste, ce qui rendait la comparaison encore plus directe. Mais dans la plupart des comptes rendus, Debussy n'est pas nommé ${ }^{16}$; parfois, le critique souligne même que dans les mélodies de Dandelot « l'accent est toujours personnel » (Chemoul-Bruyère 1938). Hoérée, après avoir étudié la partition de Dandelot, propose des considérations stylistiques qui, en considérant le langage musical plutôt que les textes choisis, ne se limitent pas à la comparaison avec le cycle de Debussy. Hoérée reconnaît des influences multiples dans l'œuvre de Dandelot (Fauré, Debussy, Ravel, Duparc). Mais s'insérer dans le sillage des maîtres de la mélodie française ne serait pas, d'après lui, un obstacle à un résultat personnel :

Le « genre français » n'est point l'apanage de quelques grands maîtres, mais le résultat de nombreux apports, d'une longue gestation [...]. Il n'y a pas que des pères, il y a aussi des fils. Le langage des grands aînés devient une tradition et je ne vois pas la raison qui empêcherait un véritable artiste de faire œuvre personnelle en parlant une langue qui a cours (Hoérée 1932, p. 387) ${ }^{17}$.

\section{LE CHOIX DES TEXTES}

Le triptyque choisi par Debussy parmi les 146 chansons de Louÿs ${ }^{18}$ a, a posteriori, une justification dramatique plutôt évidente : séduction ("La flûte de Pan »), acte sexuel ("La chevelure »), disparition de l'amour (" Le tombeau des Naïades »). A posteriori, parce que l'histoire de la composition du triptyque ne laisse entrevoir aucun plan préalable : au début, Debussy voulait composer "La flûte de Pan » (un choix très debussyste, si on pense au Prélude à l'après-midi d'un faune ou à Diane au bois), et Louÿs lui proposa "La chevelure »; les deux mélodies virent le jour presque au même moment (22 juin 1897 pour la première, 5 juillet pour la deuxième). Debussy aimait la formule du cycle de trois mélodies ${ }^{19}$, et donc il décida vraisemblablement de composer une troisième Chanson pour accomplir une œuvre unitaire

Il est aussi bien capable de pièces légères, finement ouvragées comme ses Chansons de Bilitis, que de vastes constructions comme son oratorio Pax» (Landormy 1943, p. 307).

16 En 1929, voir Chevaillier, Demarquez, Deré, Lapommeraye ; en 1930, Chevaillier, Cools, Febvre Longeray ; en 1931, A. D., Dandelot (lui-même recenseur de ses propres pièces !), Trawel ; en 1932, Capdevielle, Hoérée ; en 1933, Petit, Raugel ; en 1935, Petit, Tolleron.

17 Dans le même esprit, Imbert (1934) : «M. G. Dandelot s'est penché contre le texte, en a écouté les résonances intimes et, d'une plume sincère, a transcrit sur les portées ce qu'il entendait. Il avait sur la table l'écritoire de Debussy. L'héritage n'est pas à dédaigner pour qui sait s'en servir. M. G. Dandelot a l'habilité nécessaire ».

1893 plus 7 « non traduites » dans la première édition (1895), 146 plus 12 « non traduites » dans la deuxième (1898). Louÿs prétendait être le traducteur d'anciens textes grecs, d'où la blague de laisser les plus « osés» non traduits. Sur Louÿs traducteur et sur la blague Bilitis, voir Niederauer 1981, chap. 3.

19 Voir code 2012. 
sur Bilitis. "Le tombeau des Naïades " fut probablement un choix a posteriori par rapport aux deux mélodies qui étaient déjà prêtes : elles se trouvaient ainsi à former trois étapes d'une histoire d'amour.

Rumph a proposé d'ajouter aux raisons thématiques du choix des trois textes des raisons formelles, en soulignant qu'elles évoluent graduellement du monologue au dialogue :

The first song begins with a conventional lyric utterance, in which Bilitis recounts a tryst with her lover. In the second song, however, the lover's speech actually enters into the poem via quotation. The third song enlarges the number of speakers still further, as Bilitis quotes both another character and herself. [...] In short, songs seems to evolve in the direction of opera (Rumph 1994, p. 465) ${ }^{20}$.

Ni Koechlin ni Dandelot n'écriront leurs mélodies sur les mêmes textes choisis par Debussy pour ses Chansons de Bilitis : la confrontation directe est ainsi soigneusement évitée. D'autres compositeurs prirent - consciemment ou non - une plus grande distance encore, en écrivant des Bilitis instrumentales : D. Yzelen et Hasenfeld (pour piano), B. Crocé-Spinelli (1904, pour piano et violon), Mathé (1913, « danse grecque»), Dequin (qui, comme son recenseur Himonet [1925] le remarqua, " s'est abstenu de les traiter vocalement, s'épargnant ainsi l'aléa d'une comparaison défavorable ») et Grandjean (1920, « entr'acte symphonique »).

On constate qu'un nombre limité de poèmes a été sélectionné par plusieurs compositeurs, malgré l'abondance des choix offerts par le vaste recueil de Louÿs. Les trois textes du recueil debussyste ont été choisis au moins par un autre compositeur: "La flûte de Pan " (Debussy, Strohl, Cuvelier, Piechowska), "La chevelure " (Debussy, Strohl), «Le tombeau des Naïades » (Debussy, Cuvelier). Il est important de différencier le statut de ces choix : Rita Strohl composait ses chansons parallèlement à Debussy, alors que nous pouvons lire un désir explicite d'hommage (ou confrontation) à Debussy chez Cuvelier ou Alina Piechowska (qui publia ses Bilitis en Pologne et en France dans les années 1970) ${ }^{21}$.

Quels sont-ils les critères de sélection des Chansons de Bilitis chez Koechlin et Dandelot? Suivent-ils l'idée de progression (thématique et formelle) du triptyque de Debussy ou ont-ils d'autres conceptions du cycle?

20 Gibbons propose que la sélection faite par Debussy soit un cas illustrant ce qu'il appelle « narrative expansion » : le compositeur aurait choisi les trois points cardinaux de la première partie du cycle poétique tout en laissant le soin à la part de l'auditoire connaissant l'entièreté du recueil de Louÿs de l'entendre entre les lignes, "filling in the gaps in the poetry by using musical signifiers of the absent information " (Gibbons 2008, p. 9).

21 D'autres poèmes ont un grand succès : « Berceuse » (Strohl, Morales, Dandelot, Fiévet), « La nuit » (Strohl, Dandelot, Le Grand), "Bilitis » (Strohl, Le Grand, Piechowska), " Roses dans la nuit » (Strohl, Thiel, Cuvelier), «La pluie » (Pollet, Fayau, Cuvelier), «La lune aux yeux bleus » (Thiel, Fayau, Piechowska). D'autres ont été mis en musique par deux compositeurs : "Le sommeil interrompu ", "La quenouille " (Strohl, Dandelot), "Le serment » (Strohl, Le Grand), "Hymne à la nuit », " Chant funèbre », « [Dernière] Épitaphe » (Koechlin, Cuvelier), « Chanson », « Le réveil » (Dandelot, Cuvelier), « Lykas », « Roses dans la nuit » (Strohl, Cuvelier»), « La pluie au matin » (Langlois, Dandelot), « Chant pastoral » (Thiel, Cuvelier). 
Les Chansons de Bilitis de Koechlin sont, en ce qui concerne le choix des textes, les plus éloignées qu'on puisse imaginer de celles de Debussy : l'amour cède sa place à la mort, et l'idée de dialogue est remplacée par celle d'invocation. De plus, si Debussy ne puisait que dans la première partie du livre de Louÿs, Koechlin l'évite en faveur des trois autres.

\begin{tabular}{|lll|}
\hline « Hymne à Astarté » & III, 99 & invocation \\
«Pluie au matin » & III, 154 & mort \\
«Chant funèbre » & II, 98 & invocation + mort \\
« Hymne à la nuit » & III, 100 & invocation \\
«Épitaphe de Bilitis » & IV, 158 & mort \\
\hline
\end{tabular}

Figure 1: Textes de Louÿs sélectionnés par Koechlin (Chansons de Bilitis) et thèmes.

Il semble, donc, que Koechlin voulait créer un recueil tout à fait différent de celui de Debussy (même au plan musical, on le verra). Les poèmes de Samain qu'il choisit pour ses mélodies dédiées à Debussy n'en sont pas proches non plus : si l'amour est maintenant présent, il s'agit d'un amour raconté, et non pas vécu ; le « je » est absent de tout le recueil, sauf de la première et de la dernière mélodie. Une dimension de rêve vient ainsi encadrer toutes les narrations ${ }^{22}$.

\begin{tabular}{|c|c|c|}
\hline «L'île ancienne » & $\mathrm{I}^{\mathrm{e}}$ personne : rêve & $\begin{array}{l}\text { «Je rêve d'une île ancienne, I D'une île grecque au } \\
\text { nom d'or pur... » }\end{array}$ \\
\hline «Le repas préparé » & Discours & (Pas d'amour) \\
\hline «Le cortège d'Amphitrite» & Description & La nymphe Amphitrite se fait violer par les Tritons \\
\hline «Amphise et Melitta » & Description et dialogue & Amour paisible d'Amphise et Melitta \\
\hline «Soir païen $»$ & Description & Baiser entre Diane et Endymion \\
\hline «Rhodante » & Description & Acte sexuel entre Rhodante et un faune \\
\hline « La maison du matin » & Description & (Pas d'amour) \\
\hline «Le sommeil de Canope» & Description & Alcis embrasse Canope qui dort \\
\hline «Améthiste » & Description & Amour triste d'un faune pour une nymphe \\
\hline «Accompagnement » & $\mathrm{I}^{\mathrm{e}}$ personne : rêve & $\begin{array}{l}\text { «En un rêve, en un rêve étrange, au temps des rêves } \\
\text { | J'ai vogué sur les flots d'un océan sans grèves... » }\end{array}$ \\
\hline
\end{tabular}

Figure $2^{23}$ : Poèmes de Samain sélectionnés par Koechlin pour ses mélodies dédiées à Debussy.

Si les trois mélodies de Debussy narrent les différentes phases de l'amour vécues par le même personnage à travers des textes à fort contenu symboliste, les mélodies de Koechlin-Samain décrivent différentes scènes érotiques (la violence, l'acte sexuel, l'amour apaisé, la tristesse de l'amour non partagé) dont les acteurs sont, soit des personnages mythologiques, soit des divinités, soit des hommes. C'est comme si le

22 «Les poèmes de Samain lui permettent d'évoquer une Grèce charmante, arcadienne, toute de grâce et de légèreté ", pendant que les Chansons de Bilitis choisies par Koechlin révèlent l'autre visage de la Grèce, l'archaïsme barbare : " la Grèce ne revêt-elle pas uniquement un aspect diurne, solaire, apollinien chez Koechlin : celui-ci fait également place à la nuit, à l'ivresse dionysiaque, à la mort, à l'angoisse " (Corbier 2010, p. 338-339). Voir la section sur 1' "Hymne à Astarté de Koechlin, ou L'antidebussysme ».

23 Le texte de la dernière mélodie est d'E. Haraucourt. La première des Quatre mélodies, op. 35 sur textes de Samain était " J'ai rêvé cette nuit », mais Koechlin l'a substituée par celle de Haraucourt (sur le même sujet, la narration en première personne d'un rêve passé). 
poète en rêve survolait une Grèce imaginaire et zoomait sur des scènes "typiques ", en donnant une sorte de galerie d'esquisses, de catalogue d' " amours grecques", auxquelles s'ajoutent deux scènes domestiques (une mère qui invite la fille à préparer le souper dans "Le repas préparé ", et une autre mère qui surveille ses enfants qui jouent près de sa maison au bord de la mer dans « La maison du matin »).

Les Chansons de Dandelot empruntent à Debussy le souci d'unité dans le choix des textes, avec une attention particulière aux thèmes symbolistes tels que le rapport nuit/ jour. Le premier recueil, entièrement tiré de la première partie du livre de Louÿs (comme les trois mélodies de Debussy), explore les nuances entre l'innocence et la violence dans des scènes qui sont très connotées par leur déroulement diurne ou nocturne.

\begin{tabular}{|c|c|c|c|}
\hline « Le passant » & I, 7 & soir & $\begin{array}{l}\text { - Violence : un homme embrasse Bilitis et elle crie et pleure } \\
\text { comme si un scorpion l'avait piquée } \\
\text { - Innocence : sa mère l'embrasse aussi }\end{array}$ \\
\hline « Les contes » & I, 18 & [jour] & $\begin{array}{l}\text { - Innocence des enfants: l'amour des enfants pour Bilitis } \\
\text { est dépourvu de toute violence, leur contact est innocent }\end{array}$ \\
\hline «Le sommeil interrompu» & $\mathrm{I}, 35$ & nuit & $\begin{array}{l}\text { - Violence : l'homme s'unit à Bilitis qui s'est endormie (le } \\
\text { sommeil est imprudent) } \\
\text { - Innocence : Bilitis est comme un enfant qui ne se peut pas } \\
\text { défendre } \\
\text { - Violence qui devient innocence : après avoir crié, lutté et } \\
\text { pleuré, Bilitis s'abandonne à la tendresse de l'étreinte }\end{array}$ \\
\hline « La nuit » & $\mathrm{I}, 44$ & nuit & $\begin{array}{l}\text { - Violence : il est maintenant Bilitis a « violer » son homme } \\
\text { (qui dort) } \\
\text { - Innocence : l'amour physique est devenu un jeux }\end{array}$ \\
\hline «Berceuse » & $\mathrm{I}, 45$ & soir & $\begin{array}{l}\text { - Innocence des enfants : Bilitis chante une berceuse pour sa } \\
\text { petite fille (le sommeil est paisible) }\end{array}$ \\
\hline «Les petits enfants » & $\mathrm{I}, 17$ & jour & $\begin{array}{l}\text { - Innocence des enfants : la nudité des enfants n'est pas } \\
\text { scabreuse, les attaques des garçons aux petite filles ne sont } \\
\text { pas violence mais jeux }\end{array}$ \\
\hline
\end{tabular}

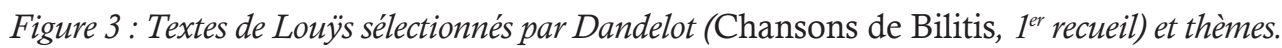

Le thème du contact physique émerge comme le fil conducteur du premier recueil. Le contact des enfants est toujours innocent, qu'il s'agisse du contact entre les enfants et Bilitis («ils courent à moi, et s'accrochent à ma tunique et prennent mes jambes dans leurs petits bras »; " ils me caressent », " ils m'embrassent sur la joue, ils posent leurs têtes sur mes seins") ou du contact des enfants entre eux (" les petits garçons jettent de l'eau sur les petites filles qui rient»). De plus, la lutte de Bilitis qui cherche à se libérer de l'étreinte du jeune homme est inutile comme le serait la lutte d'un enfant (" mais déjà il était trop tard. Et que peuvent les mains d'un enfant? »). C'est que le même geste se positionne à un différent degré sur l'échelle entre violence et innocence selon qui le pose (le baiser de la mère sert à apaiser Bilitis suite au baiser qu'elle a reçu par le jeune homme). Les insectes prennent aussi des connotations différentes (le baiser du jeune homme est violent comme le piquage d'un scorpion, tandis que les enfants, " s'ils ont pris un scarabée, ils le mettent dans ma main »). Lorsque Bilitis tombe amoureuse, le contact physique avec le jeune homme gagne le champ lexical de l'innocence, et donc de l'enfance : "Ainsi nous jouons dans la nuit ». La nuit, qui était associée à la violence, devient le temps de la jouissance amoureuse ("Chaque nuit, très doucement, je quitte la maison et je vais [...] le regarder dormir »; " première aube, ô clarté méchante, toi 
déjà ! En quel antre toujours nocturne, sur quelle prairie souterraine pourrons-nous si longtemps aimer, que nous perdions ton souvenir... ") et du sommeil paisible de la petite fille de Bilitis, enfant né d'un contact qui n'a plus rien à voir avec la violence ("Dors. Pour qu'il ne t'éveille pas, je vendrais le soleil à la mer »).

Pareillement au cycle debussyste, le premier recueil de Dandelot trace une histoire de l'expérience amoureuse de Bilitis ; la conclusion du cycle par une chanson relevant du monde de l'enfance (la seule qui ne suit pas l'ordre des chansons de Louÿs), suivant la berceuse de Bilitis à sa petite fille, veut peut-être suggérer que la fillette de Bilitis est parmi ces enfants, et donc symboliser la fusion accomplie entre les deux dimensions que les deux premières mélodies mettaient en opposition, celle de la violence du contact homme-femme et celle de l'innocence de l'enfance (et du contact mère-fille). Si la fille de Bilitis est parmi ces enfants, et donc si les deux dernières mélodies du cycle thématisent le rapport mère-fille, cela serait un lien avec le deuxième recueil, où la figure de la mère est une présence forte.

\begin{tabular}{|c|c|c|c|}
\hline «Le réveil » & $\mathrm{I}, 8$ & jour qui commence & «ma mère n'est pas éveillé » \\
\hline «La quenouille» & I, 29 & journée & $\begin{array}{l}\text { «ma mère m'a enfermée au } \\
\text { gynécée » }\end{array}$ \\
\hline «Conversation » & III, 108 & jour, projets pour la nuit & \\
\hline «Chanson» & III, 136 & $\begin{array}{l}\text { «mes cheveux sont noirs comme } \\
\text { ceux de la nuit sur la mer et mes } \\
\text { yeux bleus comme ceux du matin » }\end{array}$ & $\begin{array}{l}\text { «Le troisième était si beau que sa } \\
\text { mère ne l'embrassait sans rougir » }\end{array}$ \\
\hline «La pluie au matin » & III, 154 & jour qui commence & $\begin{array}{l}\text { «Ils apprendront mes vers, et les } \\
\text { enfants de leurs enfants » }\end{array}$ \\
\hline
\end{tabular}

Figure 4: Textes de Louÿs sélectionnés par Dandelot (Chansons de Bilitis, $2^{e}$ recueil) et thèmes.

Ce deuxième recueil est encadré par deux scènes de Bilitis qui se réveille ${ }^{24}$ : au début, elle est la jeune fille pure qui pense à la journée l'attendant à l'étable (avec une préfiguration de la courtisane qu'elle deviendra : le plaisir qu'elle prend à sucer les mamelles des chèvres deviendra bientôt le plaisir de ses amants avec les siennes) ; à la fin, elle est la vieille courtisane qui va bientôt mourir et qui va trouver la vie éternelle dans les vers qu'elle a écrits sur ses amours passés. La deuxième et la troisième mélodie comparent deux journées des deux Bilitis : la jeune fille qui s'ennuie au gynécée et la courtisane qui reçoit les avances d'un homme pour la nuit.

Les thèmes du sommeil et du réveil reviennent en ouverture du troisième recueil, entièrement tiré de la seconde partie du livre de Louÿs (celle concernant la relation lesbienne entre Bilitis et Mnasidika). Dans "La dormeuse » (II, 69), plusieurs des thèmes touchés dans les deux premiers recueils se mêlent et changent : Bilitis regarde Mnasidika qui dort au premier matin (comme elle le faisait dans « Le réveil»), mais elle ne va pas "violer » son amante après l'avoir regardée dormir (comme dans "La nuit » ou comme le faisait le jeune homme avec elle dans «Le sommeil interrompu »). Au contraire, elle souhaite que Mnasidika continue à dormir (comme elle le souhaitait 
pour sa petite fille dans la « Berceuse ») : Bilitis gagne un rôle maternel par rapport à son amante, alors qu'à la fin du premier recueil elle était mère de son enfant et, pendant le deuxième, le rôle de la mère était toujours joué par d'autres personnes (sa mère, la mère d'un de ses amants, et par extension ses ex-clients, dont les enfants apprendront la vie de Bilitis par ses vers).

Le thème de la mère sert de pivot au climax érotique des deux mélodies suivantes. Dans « Le baiser » (II, 70), Bilitis dit : « Je prendrai ta bouche dans ma bouche comme un enfant le sein de sa mère »; "L'étreinte éperdue » (II, 72), finalement, est la chanson la plus explicitement sensuelle parmi celles choisies par Debussy, Koechlin et Dandelot, et reprend plusieurs thèmes.

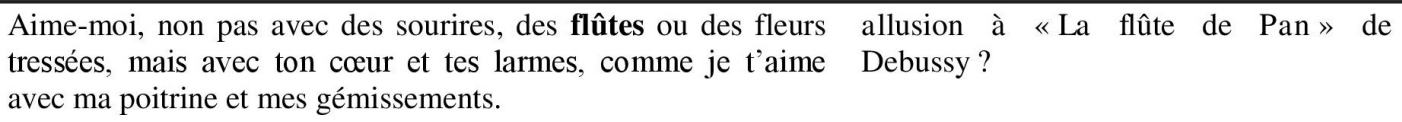

Figure $5^{25}$ : Texte de "L'étreinte perdue " de Loü̈s et thèmes.

Comme chez Debussy, la mélodie en conclusion du recueil (et du cycle entier) est un dialogue («Scène », II, 87), et comme chez Debussy, son sujet est l'amour qui se brise ${ }^{26}$.

Nous nous sommes étendus sur l'analyse des choix de Dandelot pour en montrer la complexité et le plan très précis les réglant. Abstraction faite des contacts plus ou moins directs de sa mise en musique avec celle de Debussy, on ne peut pas nier que Dandelot conçoit une œuvre organique et autonome. Ce projet dramatique complexe a été évidemment ignoré lorsqu'on en exécuta que des sélections, comme celle des Concerts Pasteloup citée ci-dessus ("Scène », « Berceuse », " Conversation »).

\section{DRAMATURGIE MUSICALE}

Une fois montrée l'idée dramatique qui a guidé le choix des textes, considérons la dramaturgie musicale des mélodies. Il s'agit d'analyser le rapport entre les textes de

25 À remarquer : la deuxième strophe, trop explicite, est coupée dans la mélodie de Dandelot.

26 Pour Rumph, les Chansons de Debussy "seems to evolve in the direction of opera " (Rumph 1994, p. 465). Dandelot accentue cette caractéristique en composant une version en duo de sa dernière mélodie, "Scène "; voir Demarquez : "'Scène', d'après Pierre Louÿs, est un dialogue dont Dandelot avait fait autrefois une mélodie fort réussie. Son arrangement en duo s'imposait: questions d'une mère grondeuse et réponses d'une fille énamourée en prennent d'autant plus de relief » (Demarquez 1935a, p. 208). 
Louÿs et les textes chantés (respect des unités syntactiques, coupures, changements), et les stratégies d'interaction entre musique et mots (présence de motifs récurrents, formes strophiques) ${ }^{27}$.

Chez Debussy, la présence d'un motif récurrent qui 1) ouvre la mélodie, 2) revient en correspondance d'un texte qui en suggère un contenu sémantique, et 3) conclut la mélodie, est un trait qui caractérise plusieurs mélodies et chacune des Chansons de Bilitis ${ }^{28}$.

En ce qui concerne Koechlin, la comparaison est simple : aucune de ses Chansons de Bilitis ne suit ce modèle. Dans un cas, "Hymne à la nuit ", le motif d'ouverture revient à la fin, écrit en 4/1 plutôt qu'en C, sans jamais avoir été entendu entre-temps (figure 6).

Dans « Pluie au matin », par contre, le motif initial se superpose (avec des modifications) au texte chanté, en créant un enjambement entre la première et la deuxième strophe (ligne en tiret dans l'exemple), mais il ne reviendra pas à la fin (figure 7).

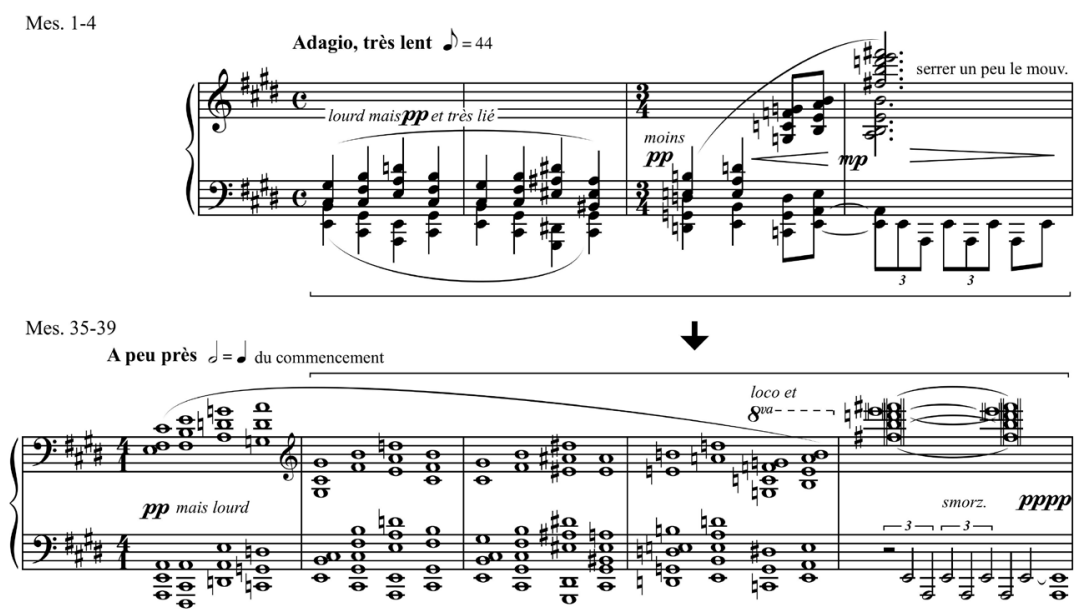

Figure 6: Charles Koechlin, "Hymne à la nuit», mes. 1-4 et 35-39 (Koechlin [1908]1923).

27 Si nous avons parlé et parlerons de «strophes ", c'est parce que Louÿs écrit toutes ses chansons en quatre parties de trois ou quatre lignes, comme si elles étaient des sonnets en prose. En fait, Louÿs avait choisi de remplacer la poésie par la prose par souci de sincérité : «La poésie est le plus pur, le plus rare, le plus difficile, le plus charmant de tous les jeux ; mais c'est un jeu » (note de P. Louÿs mentionnée par Niederauer 1981, p. 125) ; les traductions de l'Anthologie palatine lui servirent d'exercice de transformation d'idées poétiques en prose française, et les Chansons de Bilitis, conçues comme des traductions du grec, maintiennent la forme de leurs textes originels imaginaires. À ce sujet, voir Niederauer 1981, p. 125 et suivantes.

28 Puisqu'on est dans un contexte symboliste, l'interprétation sémantique n'est pas toujours univoque. Par exemple, nous trouvons peu convaincante la thèse de Rumph selon laquelle le motif instrumental de "La flûte de Pan » serait " the equivalent of a musical first-person pronoun » (Rumph 1994, p. 468). Rumph écrit que " the first return of the syrinx figure after the second stanza [...] may be heard as recalling Bilitis from the abyss of erotic annihilation toward which her intimacy with Lykas has been drawing her » (ibid., p. 469), mais, en fait, il nous semble plutôt le symbole même de l' "abyss of erotic annihilation", dès que cette figure est répétée pendant toute la troisième strophe (celle où " tour à tour » les bouches des amants "s'unissent sur la flûte ") en subissant de tourbillonnantes variations chromatiques (paradoxalement, Rumph affirme que dans cette strophe, la figure de la syrinx disparait : " when in the third stanza Bilitis' pronoun 'je' disappears within the collective 'nous', the symbol of her individual presence also vanishes ", ibid.). À propos de l'imitation du chant des grenouilles qui interrompt la dernière strophe, Rumph écrit : "When Bilitis possesses her voice we hear the music of her interior self; when that voice disappears we perceive only the sounds of the surrounding world» (ibid.) : ne pourrait-on pas plutôt parler d'un retour à la réalité après l'extase de l'ostinato qui lie les troisième et quatrième strophes? 
Mes. 1-5: Le motif initial
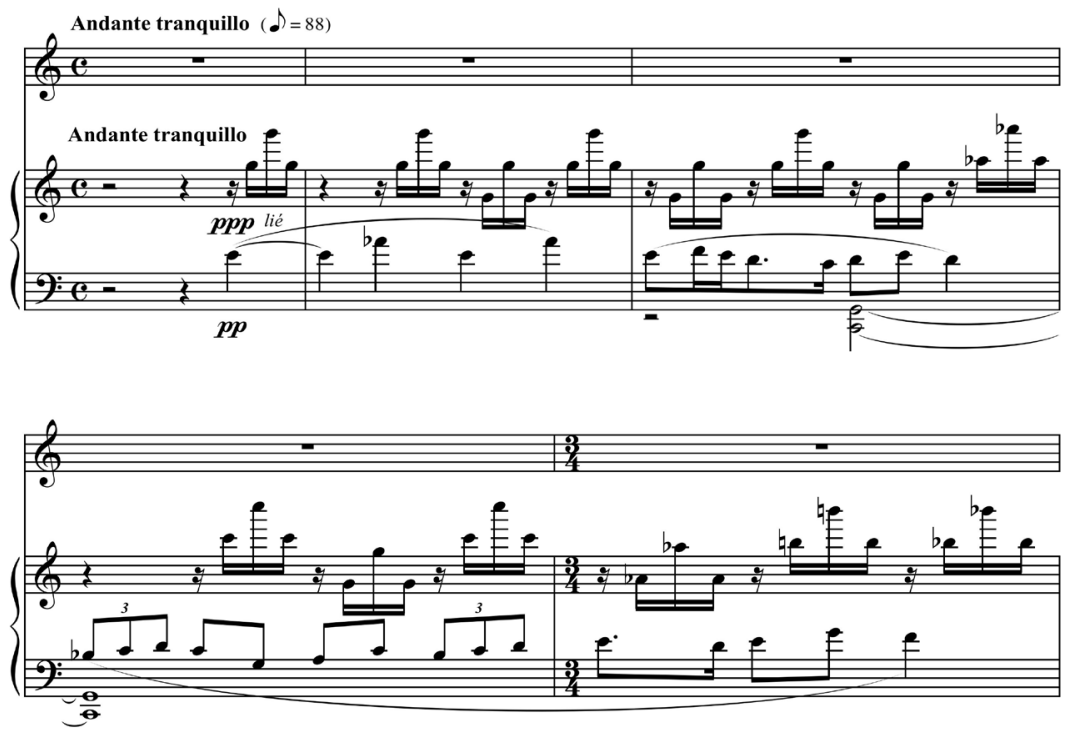

Mes. 13-17: Le motif revient entre la fin de la première strophe et le début de la deuxième
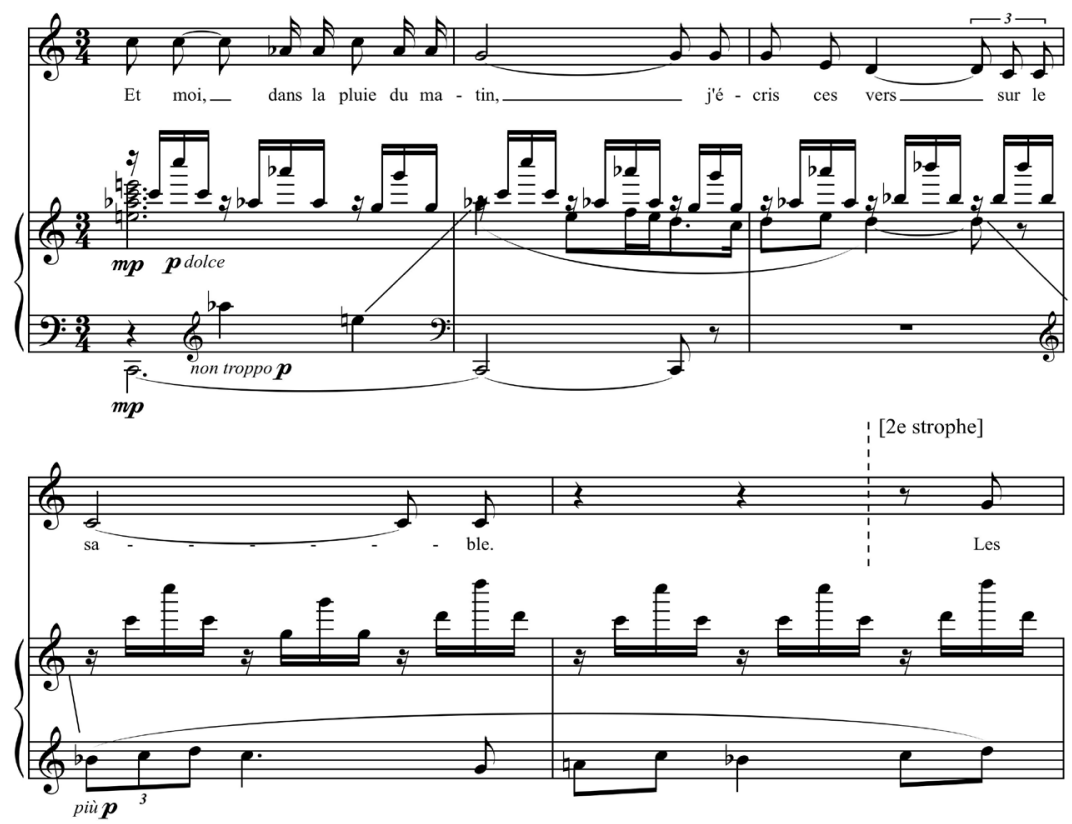

Figure 7: Charles Koechlin, "Pluie au matin», mes. 1-19 (Koechlin [1908]1923).

L' "Épitaphe de Bilitis " est encore différente. Un motif berceur est la base de l'accompagnement de presque toute la mélodie, et il disparaît dans la coda. Mais le cas le plus intéressant se trouve dans "Chant funèbre ». À partir de la mes. 21, en concomitance avec les mots "Voici qu'on a porté au tombeau Pittakos », Koechlin entame un ostinato qui est le même utilisé par Debussy dans "Le tombeau des Naïades ", un tétracorde phrygien ascendant. Est-ce une citation ? Cela est possible, puisque "Chant funèbre " fut composé en 1906-1908. Il est à tout le moins certain que Koechlin publia ses Chansons de Bilitis en 1928 (dixième anniversaire de la mort de Debussy), bien conscient que la phrase «Beaucoup sont morts que j'ai connus » 
était accompagnée par ce patron musical désormais associé aux Chansons de Bilitis debussystes (s'agit-il d'un autre cas de dramaturgie a posteriori ?) ${ }^{29}$.

C. Debussy, «Le tombeau des Naïades», mes. 1-2.

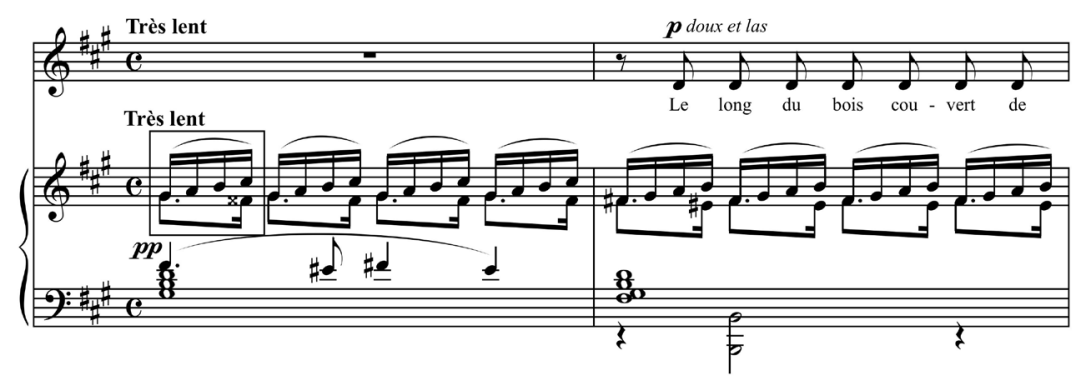

Ch. Koechlin, «Chant funèbre», mes. 20-25.
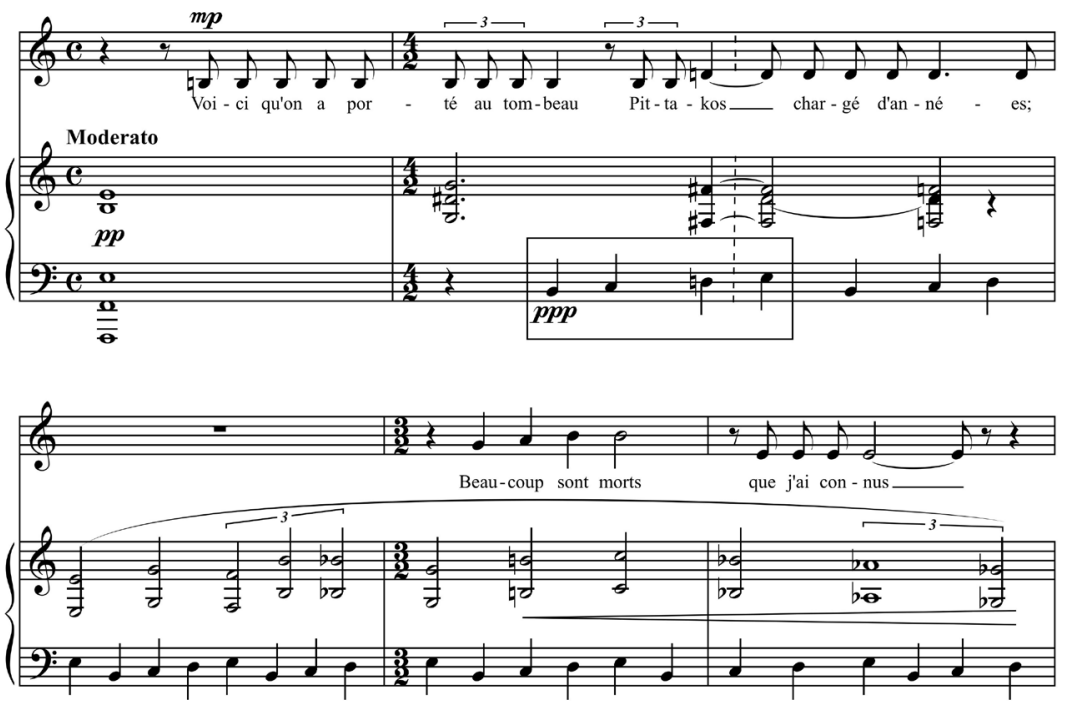

Figure 8: Claude Debussy, "Le tombeau des Naïades", mes. 1-2 (Debussy 1899), et Charles Koechlin, "Chant funèbre", mes. 20-25 (Koechlin [1908]1923).

En ce qui concerne les mélodies sur les textes de Samain, Koechlin y utilise la même variété de modèles que nous avons trouvée dans ses Chansons de Bilitis. "Amphise et Melitta » a un incipit assez debussyste : un motif au piano est répété avec la superposition de la voix la deuxième fois. Ce motif ne reviendra plus, mais le modèle de l'accord suivi par un arpège descendant régira tout l'accompagnement de la mélodie. Si dans "Le cortège d'Amphitrite " et "Soir païen » un motif initial forme la base de tout l'accompagnement (comme on l'a vu dans "Épitaphe de Bilitis "), dans "Rhodante " la répétition variée de deux motifs répartit en deux le texte de Samain : la première partie associe au statisme contemplatif du texte un motif fondé sur l'intervalle de

29 Ironie du sort, Koechlin avait coupé une phrase du texte de Louÿs qui aurait renforcé encore plus le lien entre sa mélodie et l'anniversaire de la mort de Debussy : «Celui-ci est le dixième automne que j'ai vu mourir sur cette plaine». 
seconde majeure ascendante, la deuxième partie décrit la passion du texte par des hémioles entre des accords répétés et des arpèges descendants de trois notes.

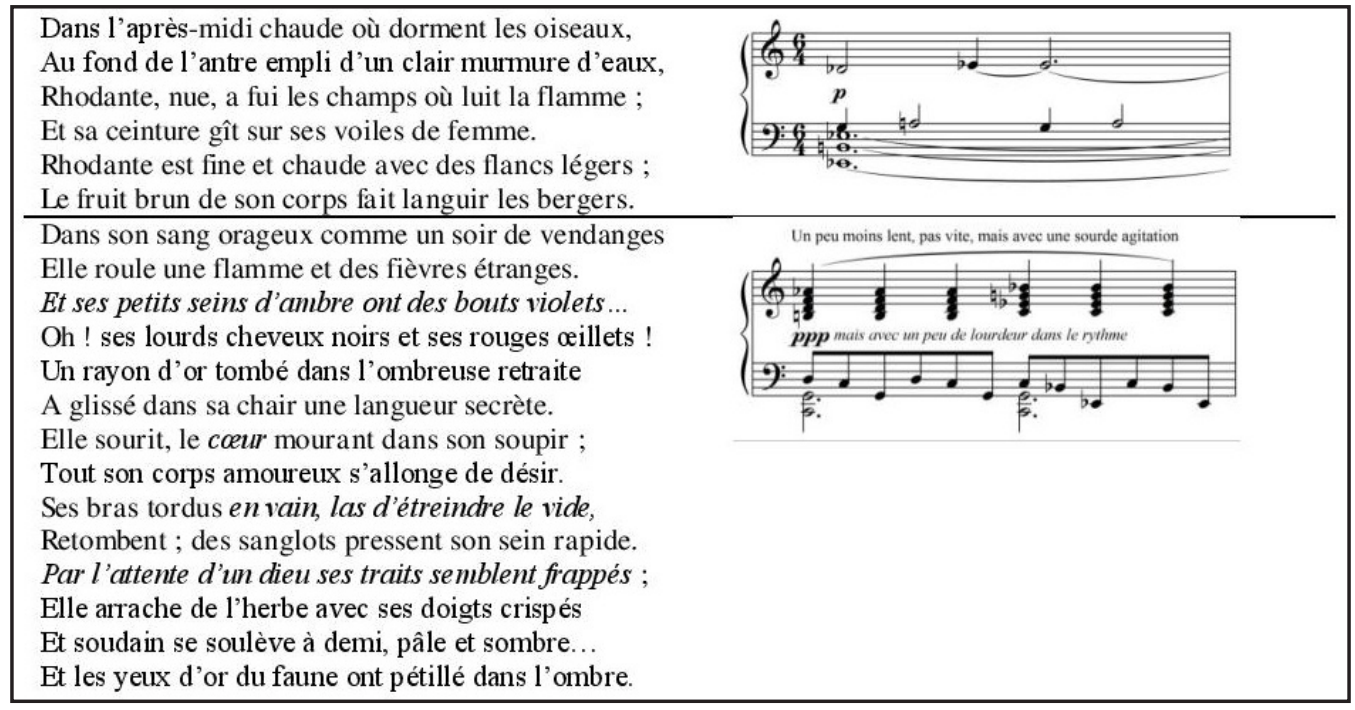

Figure 9: Charles Koechlin, "Rhodante», mes. 1 et 11 (Koechlin [1909]); les parties du texte en italique n'ont pas été mises en musique.

Dans tous les cas, nous sommes très éloignés da la dramaturgie debussyste : la pensée compositionnelle de Koechlin est fondée sur l'idée de développement, jamais sur celle de " thème objet ». Son rapport avec le texte poétique est aussi différent : il le plie à ses exigences musicales, en le coupant ou en modifiant des passages. Dans l'exemple musical 4 que nous venons de proposer, nous avons intentionnellement reproduit les vers coupés par le compositeur ${ }^{30}$. Si les textes de Samain, parfois assez longs, peuvent justifier des coupures, les Chansons de Bilitis ont par contre toutes la même taille. Debussy ne fait qu'une modification (dans "La flûte de Pan ", Debussy écrit «comme le miel ${ }^{31}$ ), expliquée par Susan Youens de la façon suivante :

Louÿs's text at the end of the first stanza/paragraph is 'comme du miel', the 'du' an element in the ' $u$ ', 'ou', and 'on' sounds that give the poem its sensuous coloring. Was Debussy's small change perhaps deliberate? The definite article is more euphonious with the l's and the rhyming '-mele' sounds that precede it (Youens 1986, p. 85$)^{32}$.

30 Pour les poèmes de Samain, nous utilisons le deuxième volume des Euvres (1924).

31 On ne doit cependant pas oublier que le titre même de cette mélodie est déjà une modification de l'originel « La flûte ».

32 Youens parle aussi d'une autre modification dans " La chevelure » : " Debussy changed from Louÿs's 'Il me dit' to 'Il m'a dit' - an improvement. Placing the account of the dream itself in the past makes it parallel to the first poem, a recollection of what had just passed, and heightens the wonderfully Symbolist tension between what is remembered and/or dreamed and the present » (Youens 1986, p. 85-86); cependant, dans l'édition critique des Chansons, il n'y a aucune trace de la variante «Il me dit». 
Koechlin, au contraire, intervient toujours sur le texte, par différents types de modifications, soit coupures, soit substitutions. Les coupures se trouvent dans "Chant funèbre " (je les indique ici en barrant les mots éliminés) : il s'agit de quelques mots (" la forêt plus éclaircie ", "je n'entends plus rien que le silence »), de la phrase qui ouvre la dernière strophe et des mots qui concluent le texte ("pleurez sur mes pas »).

Il n'y a donc pas, chez Koechlin, de coupures très étendues comme c'est le cas avec Dandelot, qui coupe des strophes entières pour des raisons de pudeur (nous avons déjà cité «L'étreinte éperdue » ${ }^{33}$ ). Le même souci de décence a poussé Koechlin à effectuer sa première substitution, le final de l' "Hymne à Astarté » ${ }^{34}$. La substitution dans "Hymne à la nuit » a probablement une raison phonétique, car le verbe "lavent » au lieu de " coulent» n'interrompt pas la succession des sons 'a' et 'e' de la dernière phrase (« Ta voix, je ne la comprends pas, mais elle me jette la tête aux pieds et mes larmes eoulent dans mes mains lavent mes deux mains »). D'autres petites substitutions n'ont aucune raison évidente ${ }^{35}$.

On trouve également des substitutions chez Dandelot ${ }^{36}$. De plus, il réorganise parfois la structure du texte pour des raisons musicales : dans "Berceuse ", il crée un refrain par la répétition de l'exhortation «Dors " associée au même patron musical, dans le but de créer une véritable berceuse ${ }^{37}$.

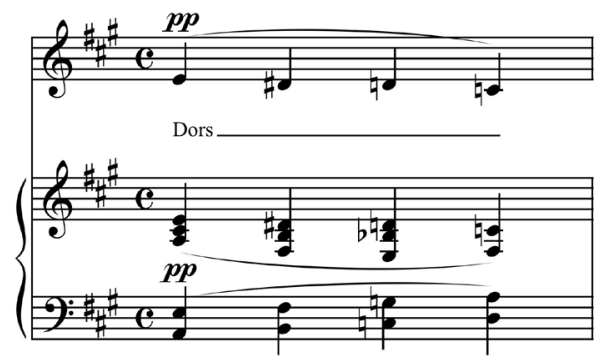

Figure 10: Georges Dandelot, "Berceuse», mes. 7 (Dandelot 1929).

Cette recherche de régularité mélodique, tout à fait antidebussyste, est évidemment justifiée par le sujet de la mélodie, et on la retrouve aussi dans "Les contes ", qui s'inspire des chansons enfantines. Mais on trouve en même temps chez Dandelot ce que Koechlin évitait, soit 1'utilisation d'un motif qui revient au cours de la mélodie en

33 Un autre cas similaire est la coupure de la quatrième strophe du "Sommeil interrompu ", quand, après son viol, Bilitis s'exclamait : «À toi, Kypris victorieuse, je consacre ces offrandes encore mouillées de rosée, vestiges des douleurs de la vierge, témoin de mon sommeil et de ma résistance ». Dans d'autres cas, comme la troisième strophe de "La dormeuse », la raison de la coupure est moins évidente.

34 «Ô Astarté irrésistible, entends-moi, prends-moi, possède-moi, ô Lune ! et treize fois, chaque année, arrache à mes entrailles la libation de mon sang arrache de mon corps bienheureux les libations sanglantes".

35 Dans « Pluie au matin » (dont le titre de Louÿs est " La pluie au matin »), " les plus âgés m'ont oubliée. C'est bien. Ils apprendront mes vers » devient « les plus âgés m'oublient. Mais ils tous sauront mes vers »; dans 1' "Épitaphe de Bilitis » (chez Louÿs " Dernière épitaphe », qui en suit deux autres), " J'ai grandi » est substitué par «Je suis née », la même formule utilisée dans les deux épitaphes précédentes.

36 Dans certains cas, les substitutions sont dues aux mêmes raisons de pudeur motivant les coupures ; par exemple, dans l'« Étreinte perdue ", " ton corps brûlant » devient le moins fort " ton corps mouvant ».

37 Au début de la dernière strophe, on trouve une variante minimale : «Fille de moi » devient « Ô fille de moi ». 
réglant la forme et en s'enrichissant de valeurs symboliques. Le cas le plus intéressant est « Le sommeil interrompu », que nous allons analyser en détails.

Concernant le rapport entre la forme poétique et la forme musicale, Debussy fait normalement entendre la coupure entre les strophes. Il utilise trois moyens : changement de la figure d'accompagnement du piano sans interruption de la ligne vocale ${ }^{38}$, bref passage instrumentale sur la même figure d'accompagnement ${ }^{39}$ et bref passage instrumentale différent (qui est habituellement le motif principal ${ }^{40}$ ). Koechlin ${ }^{41}$ et Dandelot conservent aussi, en général, la division en strophes ; pour faire cela, dans la majorité des cas ils associent un accompagnement distinct aux différentes strophes.
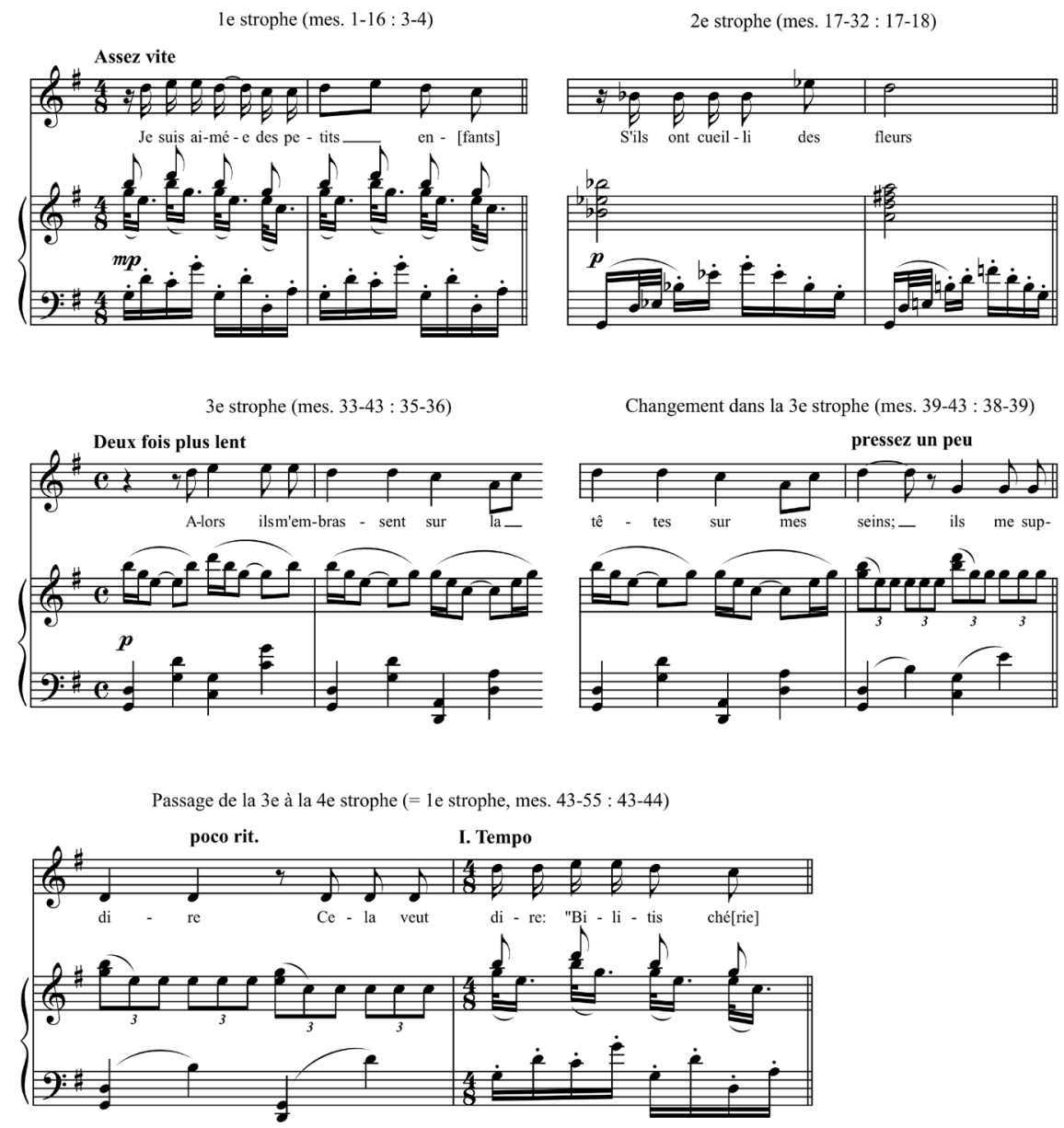

Figure 11 : Georges Dandelot, "Les contes» (Dandelot 1929).

38 «La flûte de Pan », passage entre les strophes 1 et 2, mes. 6-7 ; « La chevelure», strophes 1-2, mes. 9-10.

39 «La chevelure », strophes 2-3, mes. 15 ; « Le tombeau des Naïades», strophes 1-2, mes. 8-9 (transformation de la figure d'accompagnement) ; strophes 2-3, mes. 14-15; strophes 3-4, mes. 22 (mais il y a une coupure plus importante avant la dernière phrase de la troisième strophe, mes. 19-20).

40 "La flûte de Pan", strophes 2-3, mes. 11-12 (motif principal) ; strophes 3-4, mes. 17-18 (transformation soudaine du motif en ostinato qui soutiendra la première partie de la quatrième strophe) ; le motif du chant des grenouilles interrompt la dernière strophe aux mes. 22-24 ; «La chevelure », strophes 3-4, mes. 20 (motif principal).

41 Dans ses Chansons de Bilitis (les textes de Samain, en tant que poèmes, ne sont pas comparables à ce niveau). 
Chez Koechlin, il y a deux cas où la coupure est créée par une interruption de la ligne vocale pendant que l'accompagnement reste homogène ("Pluie au matin ${ }^{42}$ " et "Épitaphe de Bilitis ${ }^{43}$ »). Chez Dandelot, on trouve des cas plus complexes où le compositeur dépasse la division en strophes et crée d'autres divisions grâce à la récurrence de certaines formules : nous allons analyser dans le détail « Le passant » et «Le sommeil interrompu».

CAS D’ÉTUdE

Après ce survol général de la manière dont les textes poétiques ont été traités par Debussy, Koechlin et Dandelot, il est temps de regarder dans le détail ce qui unit ou bien distingue ces mélodies au plan musical. Plutôt qu'un aperçu synthétique, nous proposons d'analyser trois mélodies qui par leur dramaturgie et leur écriture nous semblent correspondre aux trois différentes attitudes possibles envers les Chansons de Bilitis après Debussy : prise de distance, influence, hommage.

"Hymne à Astarté » de Koechlin, ou L'antidebussysme (la prise de distance)

Comme nous avons déjà remarqué, deux des Chansons de Bilitis de Koechlin sont des hymnes. Le premier, à Astarté (déesse archaïque de la fertilité), ouvre le recueil ; le deuxième, à la nuit, se lie au premier puisque la nuit est considérée par Bilitis comme une force créatrice (" Ô nuit qui enfantas les dieux ! [...] comme je me sens grosse de tout ton printemps. Les fleurs qui vont fleurir cette nuit vont toutes naître de moi »).

Koechlin conçoit 1' "Hymne à Astarté " comme une prière très passionnée de Bilitis. L'aspect de prière résulte $d u$ choix d'une cantillation $d u$ texte ${ }^{44}$, mais en même temps le caractère des nuances, de l'accompagnement et du profil des phrases mélodiques est viscérale plutôt que hiératique. La première strophe peut bien illustrer cette duplicité : la voix déclame le texte sur une seule note (cantillation), introduite par un geste $f f$ du piano et accompagnée par un trémolo en crescendo (de $m p$ à ff) sur lequel elle gagne en passion (" animez et crescendo ») jusqu'au saut de quinte qui conduit à l'aigu final (encerclé dans l'exemple). Il est intéressant de noter que pour cette « prière physique » (en sujet et en réalisation), Koechlin utilise une harmonie qui n'est ni traditionnelle ni pseudoantique (modale), mais très moderne (on est en 1898), soit la gamme par tons sur do. Lors de la cadence, l'échelle change en correspondance du saut de quinte de la voix (quel geste plus cadentiel ?) : 1'accord augmenté sol-si-mi bémol fait partie de l'autre gamme par tons.

\footnotetext{
42 Voir la figure 7 : en répétant le motif initial, Koechlin crée une liaison entre les strophes 1-2, qui sont en même temps divisées par l'interruption de la ligne vocale.

43 Dans " Hymne à la nuit », Koechlin utilise les deux formes de division : strophes 1-2, mes. 7-8, changement de figure ; strophes 2-3, mes. 15-19, long passage instrumental sans modification du patron, strophes $3-4$, brève interruption de la ligne vocale.

44 En 1932, Pierre Louÿs décrira son «Hymne à Astarté» comme une « litanie monotone » (Louÿs 1990, p. 283) : la musique de Koechlin a donc bien traduit les intentions du poète (pas encore déclarées, mais sous-entendues par le texte qu'il écrit).
} 

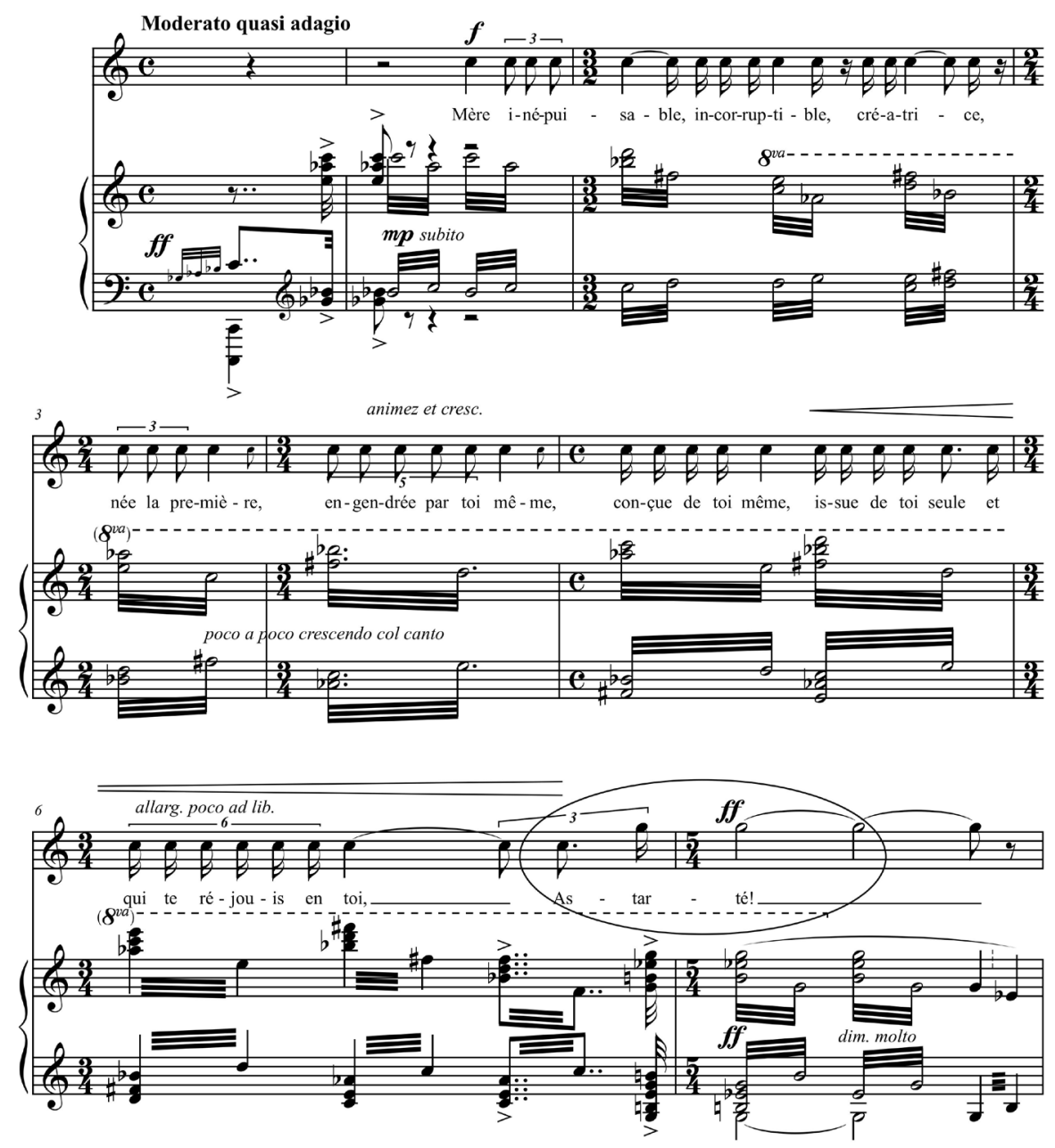

Figure 12 : Charles Koechlin, "Hymne à Astarté», mes. 1-7 (Koechlin [1908]1923).

L'idée de montée de la voix vers l'aigu en climax est maintenue dans toutes les strophes, qui se concluent pareillement avec un aigu tenu ; dans la deuxième strophe, la voix monte par degrés chromatiques (mes. 10-15). Dans la troisième strophe, la voix monte en utilisant un patron de tierce mineure ascendante répété chaque fois un ton plus haut et aboutissant à un premier aigu, mais la phrase continue, gardant l'idée de montée par tons, toujours en crescendo, vers l'aigu conclusif de la strophe (mes. 18-20). Dans la dernière strophe, le procédé de montée est encore différent : à partir de la même note, la voix atteint des notes qui sont toujours un demi-ton plus aigu que la précédente (mes. 27-33). 

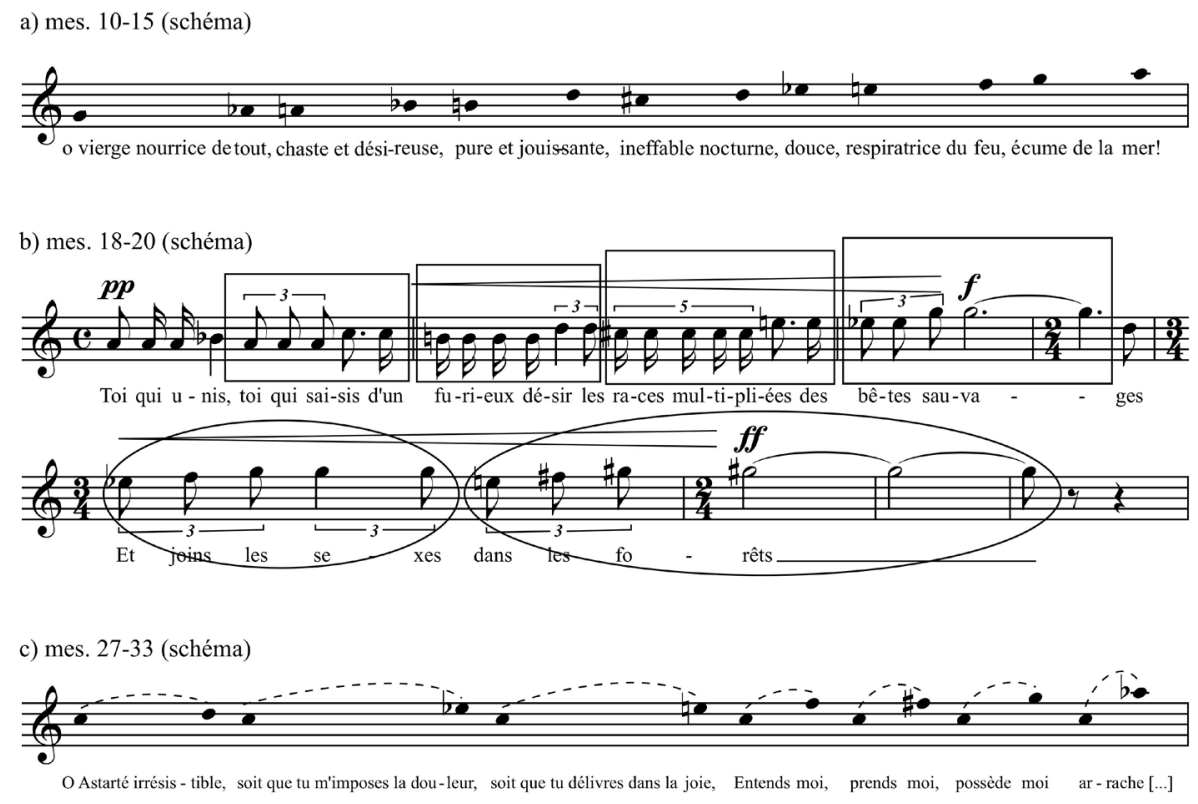

Figure 13 : Charles Koechlin, "Hymne à Astarté» (Koechlin [1908]1923), schéma de la mélodie chantée.

Il s'agit, donc, d'une conception de l'écriture vocale très directionnelle, schématique et impersonnelle (cantillation), et en même temps très extérieure, pas du tout intime, mais viscérale (passion). La "violente extase de l'adoratrice " se traduit aussi par le timbre vocale, très différent du timbre debussyste en raison du registre : de $m i_{3}$ à $s i_{4}$, tandis que la Bilitis de Debussy s'exprime entre $s i_{2}$ et $f a \sharp_{4}$ (cette note n'apparaissant que deux fois en correspondance des seuls deux $f$ du recueil, dans les climax de "La chevelure » [mes. 1845] et du « Tombeau des Naïades » [mes. 25]).

Comme Christophe Corbier l'a montré, les Chansons de Bilitis sont un des rares exemples où Koechlin choisit de mettre en musique « la face nocturne de l'Antiquité » (Corbier 2010, p. 339-342), barbare et dionysiaque ; la Bilitis debussyste fait, par contre, partie de cet « hellénisme français » (ibid., p. 332-336) que Koechlin privilégia dans ses autres compositions à sujet grec. Si, comme le remarqua François de Médicis, dans les Chansons de Bilitis de Debussy et de Koechlin "l'écriture musicale [...] est complètement différente » (De Médicis 2010, p. 213, note 2$)^{46}$, c'est surtout parce que la Grèce qu'ils évoquent n'est pas la même. Les mélodies sur les textes de Samain sont alors les « chansons de Bilitis » de Koechlin davantage que ses Chansons de Bilitis, bien que le compositeur précise son souci d'être le moins debussyste que possible :

45 Il est significatif qu'à cet aigu, qui correspond au sommet du rêve de l'amant de Bilitis (« ou que tu entrais en $m o i »)$, le piano soutienne la voix par un Tristanakkord (sol\#-si-ré-fa\#).

46 Dans cette étude, on peut lire une analyse d' "Améthiste », dont le sujet (fin de l'amour et du monde antique) est mis en parallèle au "Tombeau des Naïades " : un autre indice du fait que les mélodies sur les textes de Samain sont les "chansons de Bilitis " de Koechlin davantage que ses Chansons de Bilitis. Wenk (1976, p. 193) met aussi en relation «Le tombeau des Naïades » avec "Colloque sentimental » qui conclut Fêtes galantes ; l'ambiance hivernale partagée par les deux mélodies est absente d' "Améthiste ».) Douche (2010) analyse " Accompagnement » comme exemple du style moyen des mélodies du Koechlin d'avant-guerre. 
C'est plutôt l'atmosphère de mon troisième [recte: quatrième] recueil ("L'île ancienne ", etc.) sur des poèmes de Samain, qui offre certaine parenté avec celle du sentiment debussyste : mais je pense qu'il y a là moins d'influence réelle de Debussy, que d'analogie due au caractère même des poésies de Samain, au charme lumineux et subtil qu'il fallait obtenir ${ }^{47}$.

"Le passant» de Dandelot, ou Le pseudodebussysme (l'influence)

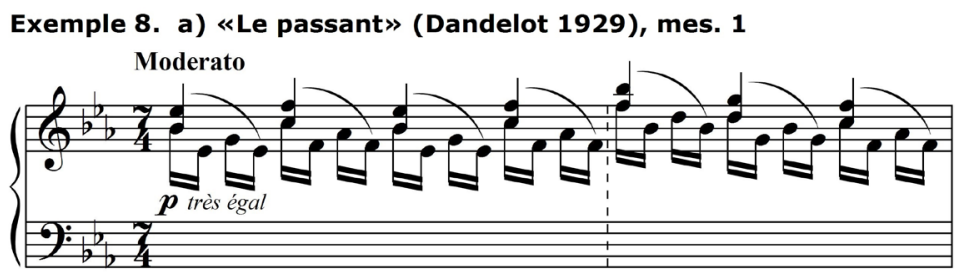

b) «La chevelure» (Debussy 1899), mes. 1

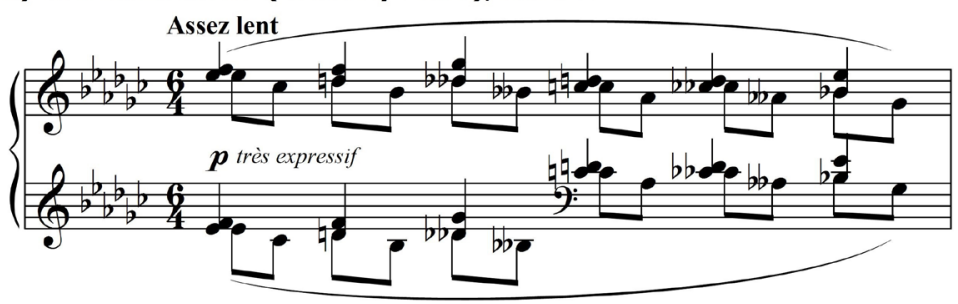

Figure 14 : Georges Dandelot, "Le passant", mes. 1 (Dandelot 1929), et Claude Debussy, "La chevelure», mes. 1 (Debussy 1899).

Au premier coup d'œil, la partition du " Passant " provoque une impression de déjà-vu. La sensation d'un calque debussyste est pourtant rapidement démentie. La ressemblance avec le geste initial de «La chevelure » n'est que très superficielle, au plan de l'écriture et de la dramaturgie : loin d'être un motif qui revient au cours de la pièce en des moments significatifs, l'arpège de Dandelot est une formule d'accompagnement par triades majeures ou mineures qui s'étend sur presque l'entière mélodie et redouble à l'octave aiguë la ligne vocale. En fait, ce procédé est aussi une caractéristique de «La chevelure » debussyste : dans la première et la dernière strophe, la ligne vocale est redoublée à l'unisson et à l'octave (grave) par le piano ${ }^{48}$ (figure 15).

Les ressemblances ne s'arrêtent pas ici. On pourrait décrire "La chevelure " comme une succession de phrases mélodiques du piano (de différentes longueurs, redoublées ou pas par la voix) basées sur la répétition interne et enchaînées l'une à l'autre (figure 16). L'idée des brèves formules mélodiques combinées et récurrentes deviendra le principe compositionnel du " Passant».

47 Lettre de Koechlin à Henri Sauguet, 9 mai 1943, in Koechlin (1982, p. 115), citée par Douche (2010, p. 186-187).

48 La voix chante aussi sur les mêmes notes du piano dans « La flûte de Pan », mes. 5, et, d'une façon plus indirecte, sur les deux premiers temps des mes. 13-14. 
Exemple 9. a) «Le passant» (Dandelot 1929), mes. 2-3

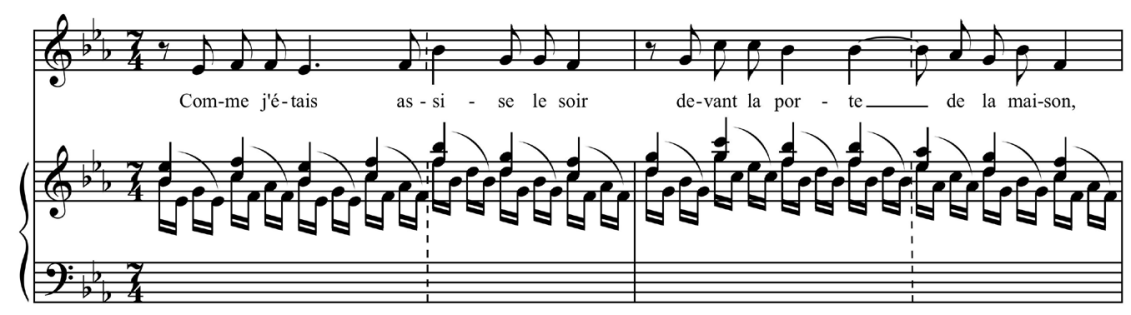

b) «La chevelure» (Debussy 1899), mes. 2-4

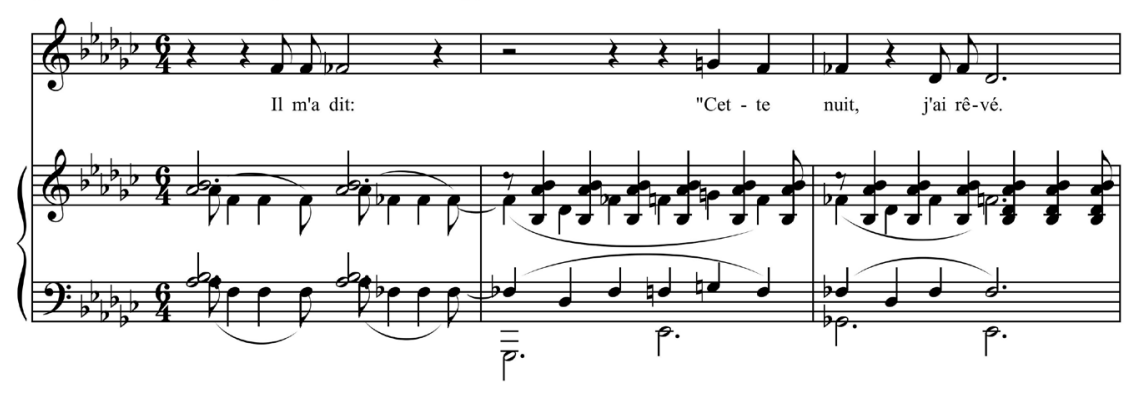

Figure 15 : Georges Dandelot, "Le passant", mes. 2-3 (Dandelot 1929),

et Claude Debussy, "La chevelure ", mes. 2-4 (Debussy 1899).

Mes. 1, répété à la mes. 20 ; la première partie conclut la pièce (mes. 25-25)

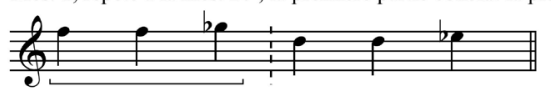

Mes. 2-3, répété aux mes. 4-5
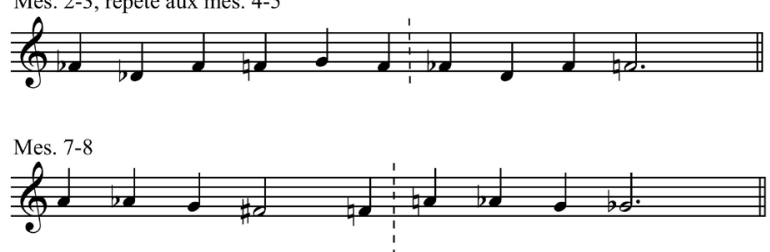

Mes. 10-11 (le patron est une variante de celui du motif initial)
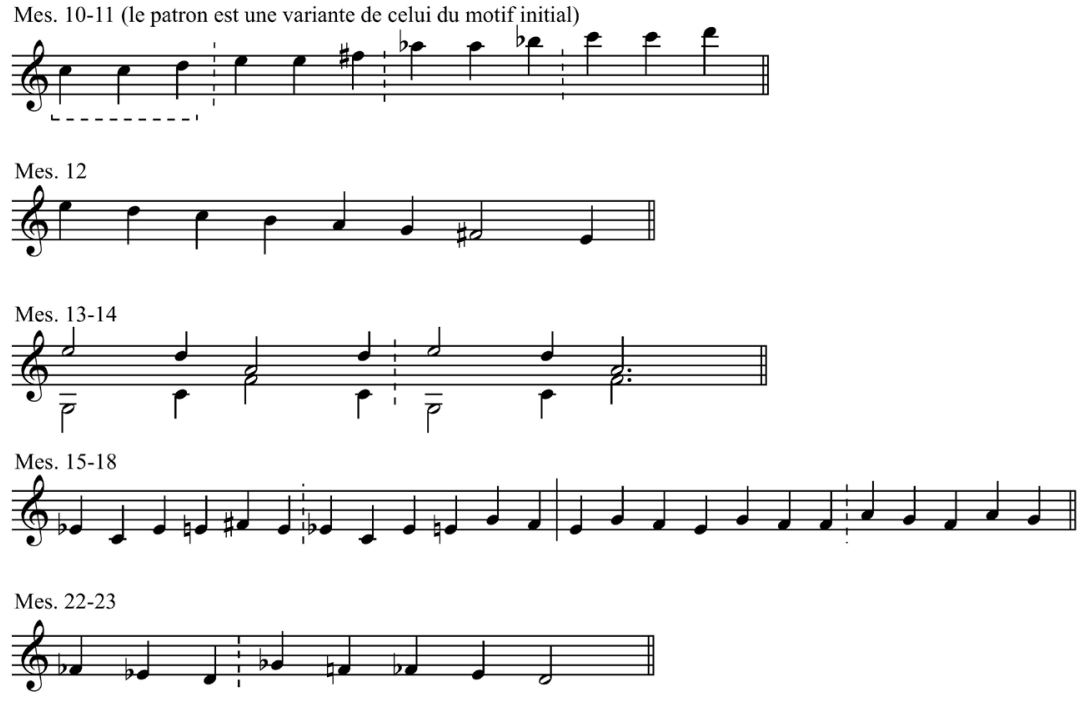

Figure 16: Claude Debussy, "La chevelure» (Debussy 1899), schéma des mélodies du piano. 


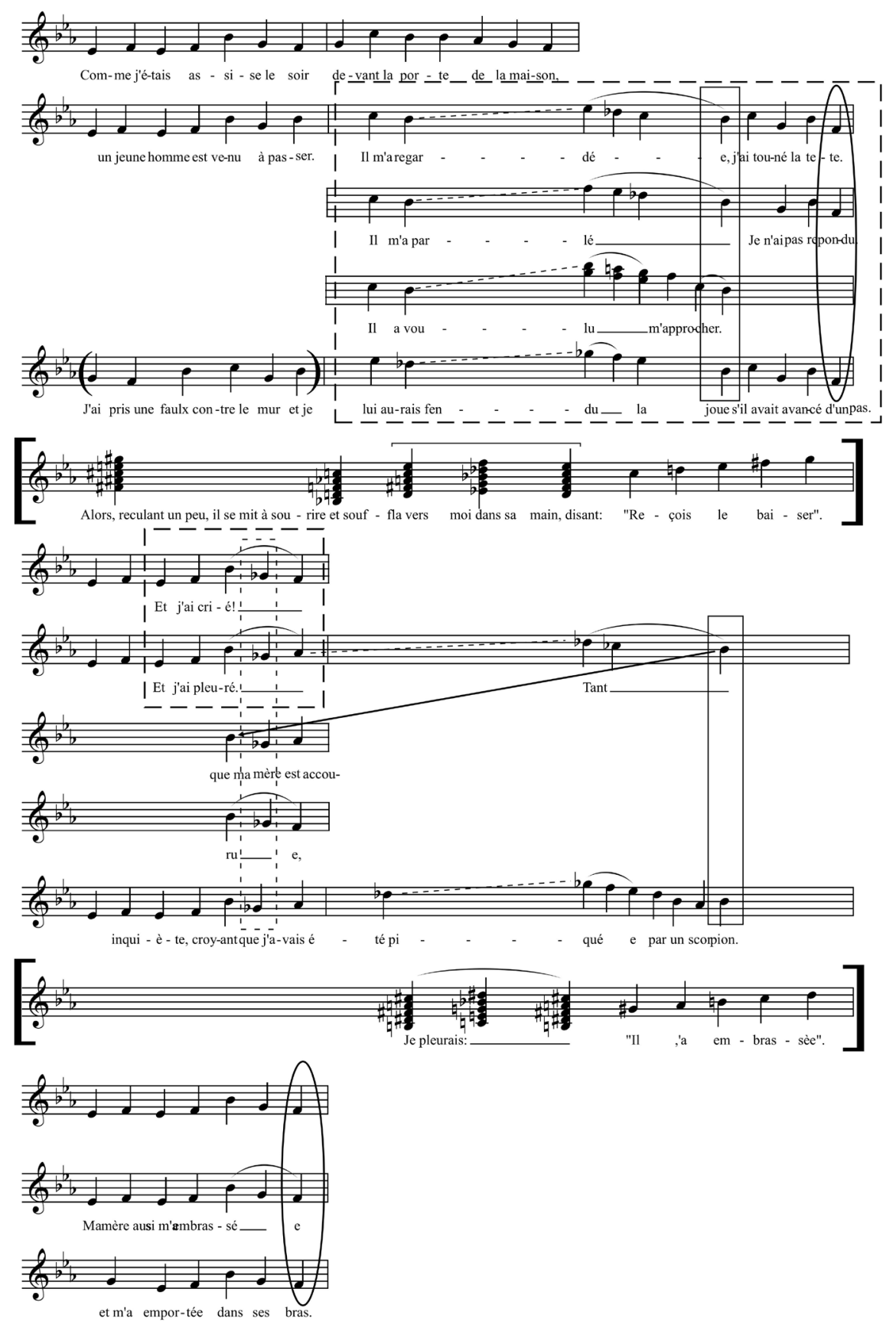

Figure 17: Georges Dandelot, "Le passant»(Dandelot 1929), schéma paradigmatique des mélodies.

Il est particulièrement intéressant que Dandelot traduise les anaphores de Louÿs par des anaphores musicales (v. les deux sections encadrées dans la figure 21). "Il m'a regardé ", " Il m’a parlé ", " Il a voulu m’approcher " utilisent la même formule mélodique, mais il ne s'agit pas d'une simple répétition, puisque la formule 
est poussée chaque fois un peu plus vers l'aigu (lignes en tiret dans l'exemple) ${ }^{49}$ : ainsi, le climax des actions du jeune homme (regarder $>$ parler $>$ approcher) est exprimé par des moyens musicaux. Dans la phrase suivante, " je lui aurais fendu la joue s'il avait avancé d'un pas ", la même formule est encore utilisée (mais sur une hauteur de départ différente ; la note sur laquelle se termine la descente est cependant toujours si bémol ${ }^{50}$ ). En fait, " lui » occupe le même axe paradigmatique que " il » dans les phrases précédentes, dès qu'il s'agit du degré successif du climax des actions du jeune homme (>avancer, qui ne se réalise pas).

L'autre anaphore réalisée musicalement par Dandelot concerne la réaction de Bilitis aux avances du jeune homme : "et j'ai crié, et j'ai pleuré ». Comme dans les cas précédents, Dandelot se sert du mélisme. Le mélisme sera exploité aussi pour les deux actions de la mère (" ma mère est accourue ", " ma mère aussi m'a embrassée "), qui répondent aux réactions de Bilitis et servent de contrepoids aux actions du jeune homme. Il est évident que Dandelot a un souci de régularité, que nous avons déjà remarqué dans «Berceuse » ou "Les contes», et qui est totalement étranger à Debussy : dans "La chevelure ", en particulier, les répétitions des figures mélodiques du piano ne correspondent jamais à des anaphores textuelles. De surcroît, l'usage du mélisme est rare chez Debussy, qui normalement assigne une note à chaque syllabe. Dans les Chansons de Bilitis, il y a pourtant un passage où un bref mélisme descendant est répété et crée une sorte de carrure ; un modèle exceptionnel que Dandelot a transformé en règle pour son « Passant ${ }^{51}$ ?

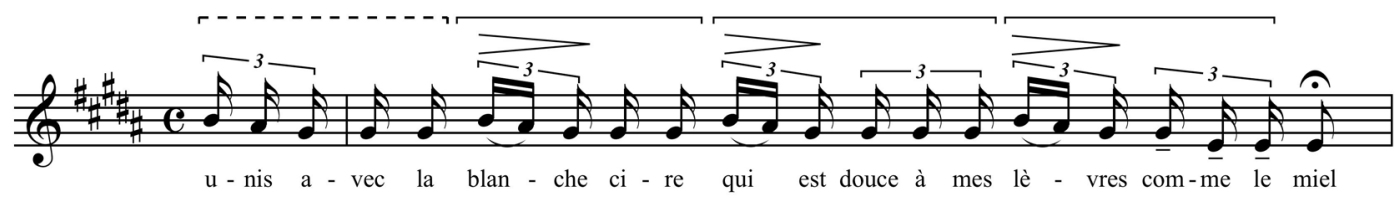

Figure 18: Claude Debussy, "La flûte de Pan ", mes. 6 (Debussy 1899).

En conclusion, dans "Le passant ", Dandelot exploite certains traits du style debussyste mais d'une façon que Debussy n'aurait jamais utilisée : la ligne vocale redoublée devient règle, le motif qui ouvre "La chevelure » devient une figure d'accompagnement, la répétition d'un mélisme acquiert un rôle dramatique prépondérant, la récurrence d'une mélodie se transforme en technique de combinaison de cellules mélodiques. Bref, des ingrédients qu'on peut retrouver dans les Chansons de Debussy mènent ici à un produit tout à fait non debussyste.

49 Pour le troisième saut, Dandelot donne la possibilité de chanter sol au lieu de si bémol.

50 La fin de la descente mélodique du mélisme sur « tant » s'enchaîne directement à la phrase suivante : dans l'exemple, nous avons montré à l'aide d'une flèche qu'il s'agit du même si bémol.

51 Il n'y a que deux autres mélismes dans les Chansons de Debussy, les deux constitués par deux notes ascendantes au début d'un triolet : "La flûte de Pan ", mes. 3, et « La chevelure ", mes. 12 (Dandelot utilisera la même formule dans "Le sommeil interrompu », aux mes. 24 et 25). L'autre cas où la même syllabe est chantée sur deux notes est en réalité un portamento ("Le tombeau des Naïades », mes. 25). 
Si l'incipit du « Passant » donnait l'impression d'un déjà-vu, celui du « Sommeil interrompu " peut provoquer chez les debussystes les plus sensibles une onde de choc : est-ce le fantôme du maître qui revient ? En vérité, comme c'était le cas dans "Le passant ", l'impression de calque debussyste s'affaiblit lorsque, suite au premier coup d'œil, on passe à l'écoute et à l'analyse. Contrairement au " Passant ", ici Dandelot ne rend pas règle ce qui, chez Debussy, était exception, mais il utilise des moyens debussystes comme Debussy lui-même l'aurait fait. Il s'agit notamment de la dramaturgie du motif principal, de la structure formelle basée sur la duplication et de l'harmonie à accords parallèles.

La mélodie s'ouvre par deux mesures au piano exposant ce que chez Debussy nous avons nommé le motif principal (voir la section sur la "dramaturgie musicale ») : un geste musical très reconnaissable au sujet de la texture et du profil qui revient au cours de la pièce en s'enrichissant de connotations symboliques par sa superposition au texte chanté. Il faut dire que, si le procédé de Dandelot est sans doute un calque debussyste, ses caractéristiques d'écriture ne le sont pas : il ne correspond à aucune des textures auxquelles Hepokoski (1984) réduit les «formulaic openings » chez Debussy (il n'est ni un "monophonic opening " ni un "modal/chordal opening » tout court), et son harmonie est, comme dans "Le passant ", basée sur la triade (le Tristanakkord incomplet de la première mesure peut rappeler l'incipit de " En sourdine » des Fêtes galantes, mais sa résolution tonale en sol mineur est décidemment « hors style»).

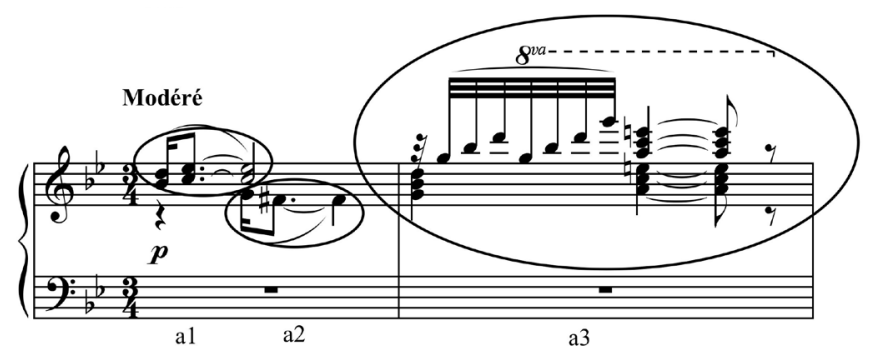

Figure 19: Georges Dandelot, "Le sommeil interrompu», mes. 1-2 (Dandelot 1929).

Dans les mesures suivantes, ce motif révèle sa nature de «introductory sequence/ expansion": après une "fermata or grand pause " (fin de la mes. 2), il se prête à une "varied repetition" (Hepokoski 1984, p. 48-50). Le motif est donc traité comme un " thème objet ", répété davantage que développé. En particulier, on peut diviser ce motif ( $a$ ) en trois composantes (voir $a 1, a 2$ et $a 3$ dans la figure 22) qui reviennent tout au long de la mélodie, séparément ou combinées, à coté de deux autres formules d'accompagnement ( $b$ : accords tenus, $c:$ accords arpégés). 


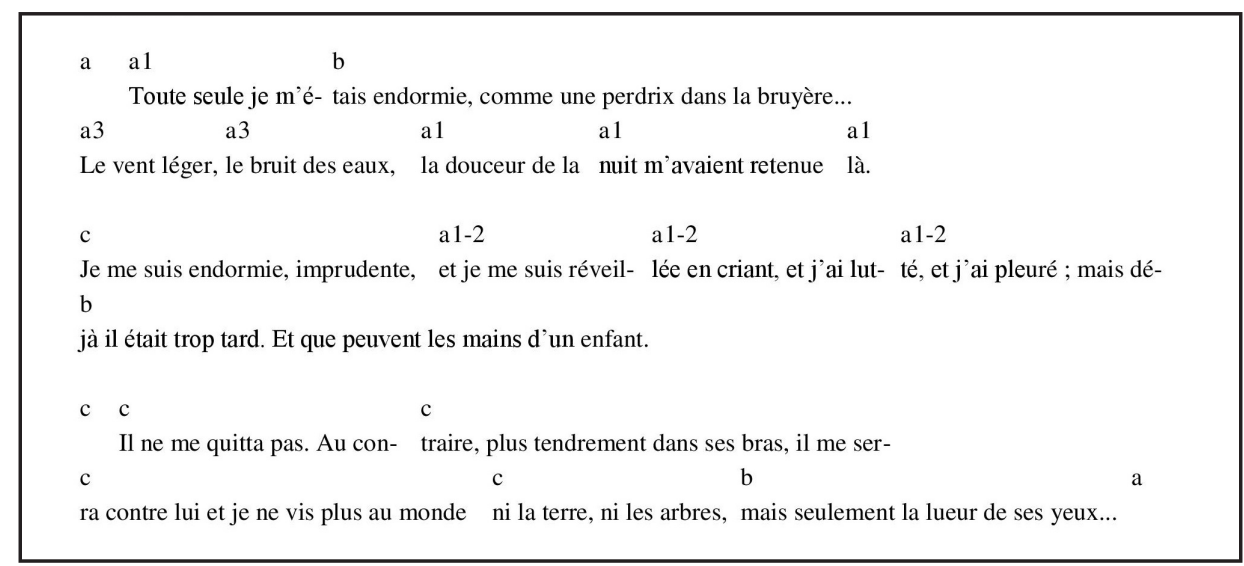

Figure 20 : Georges Dandelot, "Le sommeil interrompu ", découpage musical et texte.

Une première considération concerne $a 3$ : cette partie du motif principal, qui au début n'était qu'un arpège rapide et aigu entre deux triades mineures, se charge d'une valeur sémantique lorsqu'elle est associée au " vent léger » et au "bruit des eaux » (une valeur sémantique pas univoque, donc, selon la meilleure tradition symboliste). Quant à la première mesure du motif principal (a1-2), elle reviendra poussée à l'aigu et bâtie sur la gamme par tons : elle est maintenant un véritable " cri (a1) + sanglot (a2) ». La troisième strophe utilise surtout la formule $c$, que Dandelot enchaîne en explorant, d'une façon très debussyste, les possibilités de la duplication ${ }^{52}$.

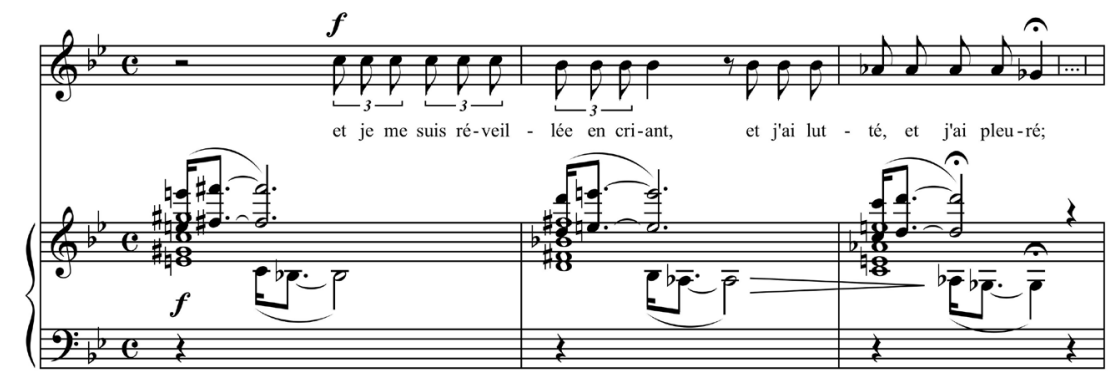

Figure 21 : Georges Dandelot, "Le sommeil interrompu», mes. 14-16 (Dandelot 1929).

52 Aux mes. 23-25, nous avons éliminé les enharmonies afin de rendre la lecture de l'exemple plus immédiate. 

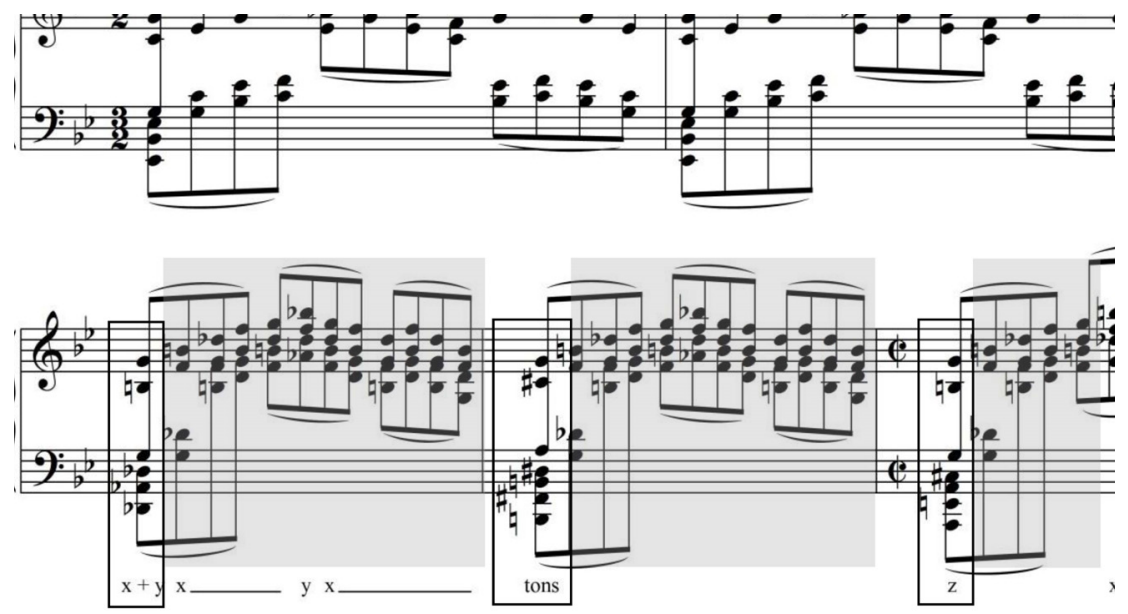

Figure 22 : Georges Dandelot, "Le sommeil interrompu», mes. 21-25, piano (Dandelot 1929).

Si la mes. 22 est une duplication exacte de la précédente, le processus de duplication protagoniste des mes. 23-25 est plus complexe. La mes. 24 est une copie de la précédente (en gris) sauf que pour l'accord initial (encadré : à la mes. 23, il était la combinaison des accords $x$ et $y$ qui vont former la suite de la mesure ; à la mes. 24, il s'agit d'un accord par tons). La mes. 25 commence selon la même règle (changement de l'accord initial et duplication de l'arpège suivant), mais après quatre croches, il y a un changement de la partie dupliquée : la mesure se termine par l'accord $x$ en profil ascendant ${ }^{53}$.

Il serait faux de penser que le résultat de cette composition modulaire soit une pièce saccadée. Dandelot, comme Debussy, est très attentif aux pivots entre les différentes formules d'accompagnement. De plus, il ne s'agit pas seulement pour le compositeur de rendre les transitions floues, mais aussi d'organiser un parcours harmonique de longue haleine ; le schéma suivant illustre l'exploitation par Dandelot de l'effet parenthèse qu'on peut rencontrer maintes fois chez Debussy ${ }^{54}$. La figure 23 montre que la deuxième strophe (en gris) est une section par tons encadrée par deux cadences parfaites qui ont en commune la même basse mi bémol-si bémol (la première fois dans le rôle de $\mathrm{V}$, la seconde dans le rôle de I). La transition entre la succession d'accords parallèles de septième et les accords par tons se fait par deux notes communes (encerclées). Si la première strophe se terminait avec une descente chromatique de la basse, la deuxième se déroule entièrement sur une descente de la basse par tons (avec demi-tons dans les transitions entre une gamme par tons et l'autre), de si bémol à si bémol ${ }^{55}$.

53 Si l'interruption de la duplication est quelque chose qu'on peut trouver souvent chez Debussy, le cas d'une duplication exacte (ou camouflée par l'enharmonie) mais avec l'incipit varié est un procédé qui ne paraît pas dans la rigoureuse classification des stratégies de duplication chez Debussy établie par Bourion (2011).

54 Voir par exemple la première section de "La cathédrale engloutie », mes. 1-15 : les mes. 7-13 sont une espèce de parenthèse qui interrompt la descente de la basse qui accompagne le geste initial et qui se poursuit à la mes. 14 .

55 On pourrait dire que la descente commence du do, qui fait partie du premier accord et est joué comme appogiature du si bémol selon le modèle $a 2$; en fait, la voix chante une gamme par tons descendante de do 


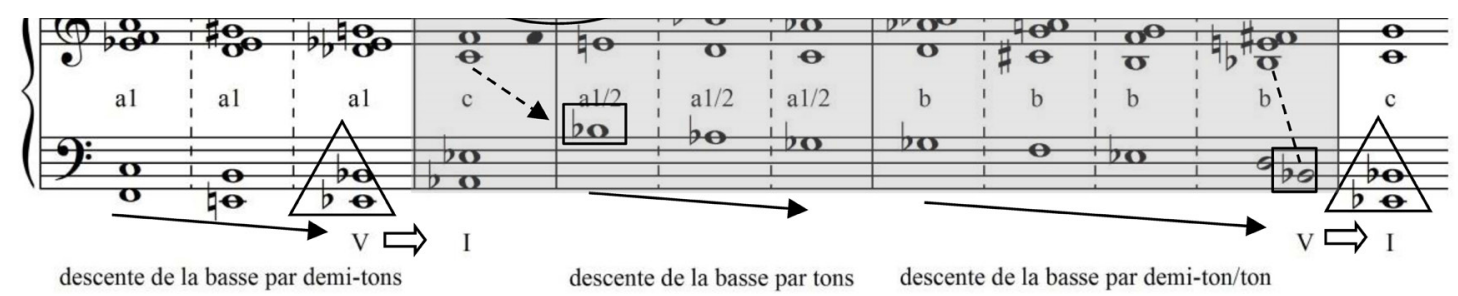

Figure 23 : Georges Dandelot, "Le sommeil interrompu », mes. 8-25 (Dandelot 1929), schéma de la partie du piano.

En résumant, une série d'indices laisse penser que Dandelot voulait créer un " faux » : la gestualité, la conception dramatique et formelle, et la recherche dans le champ de la duplication suivent ouvertement le modèle debussyste. Il y a aussi des particularités presque souterraines mais très révélatrices : si dans « Le passant », nous avons remarqué une utilisation décidemment hors style du mélisme, dans "Le sommeil interrompu ", nous n'avons que deux brefs mélismes de deux notes ascendantes au début d'un triolet, exactement comme dans les Chansons de Bilitis de Debussy ${ }^{56}$. S'agit-t-il là d'un hommage ou d'une exploitation de moyens considérés valables pour créer un ouvrage personnel ? En effet, à l'origine de l'histoire des Chansons de Bilitis, Pierre Louÿs se prétendait le traducteur d'une imaginaire poétesse ; 20 ans après, Georges Dandelot, en signant du faux Debussy, poursuivrait ce jeu de miroirs où modèles réels, modèles inventés, création personnelle, exploitation et blagues se confondent et nous confondent.

\section{CONCLUSION}

Une histoire exhaustive de l'imaginaire grec dans la musique française de la première moitié du xx $\mathrm{x}^{\mathrm{e}}$ siècle reste encore à écrire. L'intérêt pour la tragédie antique (sous forme de musiques de scène ou d'opéras), les nouveaux livrets à sujet hellénique ainsi que les études sur la musique grecque (de premier plan dans ce courant culturel est la figure de Théodore Reinach) forment un cadre de référence qui nécessite d'être pris en compte afin d'évaluer en profondeur la place occupée par Bilitis dans l'histoire de la musique.

Dans l'étude proposée ici, nous avons préféré nous concentrer sur la question "Comment Bilitis après Debussy? ", plutôt que sur la plus vaste " Pourquoi Bilitis ?". L'accent a été mis sur l'acte de s'insérer ouvertement dans les traces d'un musicien en cours de canonisation plutôt que sur la signification plus largement culturelle sous-tendant ce choix. Nous avons préféré analyser l'intertextualité en termes d'influence - pour ainsi dire - ex persona, plutôt qu'en termes de Zeitgeist.

à sol bémol, un peu décalée de celle de la basse (voir la figure 21). Par contre, le do entre le ré et le si bémol final n'est pas écrit, le si bémol étant un renversement de l'accord final.

$56 \quad$ Voir la note 51. 
Les exemples présentés - loin d'être exhaustifs - ont été jugés représentatifs d'une variété d'attitudes possibles au moment de la confrontation avec un exemple encombrant. L'analyse des stratégies compositionnelles employées par Koechlin et Dandelot (ce dernier ayant été présenté au public parisien en tant que compositeur précisément par ses Chansons de Bilitis : un baptême de feu à très haute température) a aussi permis d'approfondir la spécificité de la dramaturgie musicale et de la technique debussystes.

Bilitis, comme elle-même l'affirme dans l'épitaphe closant le recueil de Louÿs, est intrinsèquement polymorphe : "J'ai grandi sur la terre des nymphes ; j'ai vécu sur l'île des amies ; je suis morte sur l'île de Kypris ». Plusieurs compositeurs ont voulu la porter sur leur île. Mais il semblerait, cependant, que c'est uniquement grâce à l'île visitée avec Debussy que, pour les musicophiles d'aujourd'hui, « [son] nom est illustre et [sa] stèle frottée d'huile».

\section{BIBLIOGRAPHIE}

A. D. (1931), Compte-rendu du concert à l'École normale de musique (février 1933), Le Monde musical, $42^{\mathrm{e}}$ année, $\mathrm{n}^{\circ} 2$, p. 63.

Anonyme (1935), "Les travaux d'été des compositeurs ", Le Monde musical, 46e année, n 8-9, p. 273-275.

Anonyme (2009), "Catalogue des œuvres de Rita La Villette », Rita Strohl. Une personnalité mystérieuse, un compositeur étonnant, blog, http ://ritastrohl.artblog.fr/r27477/Catalogue-des-Oeuvres-etablipar-l-artiste, consulté le 18 janvier 2017.

Belvianes, Marcel (1933), Compte rendu du concert de la Société nationale (11 février 1933), Le Ménestrel, $95^{\mathrm{e}}$ année, $\mathrm{n}^{\circ}$ 7, p. 70, http://gallica.bnf.fr/ark:/12148/bpt6k5617703j.item, consulté le 18 janvier 2017.

Bertrand, Denyse (1936), Compte rendu des Concerts Pasdeloup (8 février 1936), Le Ménestrel, $98^{\mathrm{e}}$ année, $\mathrm{n}^{\mathrm{o}}$ 7, p. 52, http://gallica.bnf.fr/ark:/12148/bpt6k5617361m.item, consulté le 18 janvier 2017.

Bourion, Sylveline (2011), Le style de Claude Debussy. Duplication, répétition et dualité dans les stratégies de composition, Paris, Vrin.

Capdevielle, Pierre (1932), Compte rendu du Festival Georges Dandelot à l'École normale de musique, (février 1932), Le Monde musical, 43e année, nº 2, p. 63.

Carol-Bérard (1925), « Festival Betove », Le Courrier musical, $27^{e}$ année, n 19, p. 541.

Cathé, Philippe, Sylvie Douche et Michel Duchesneau (dir.) (2010), Charles Koechlin, compositeur et humaniste, Paris, Vrin.

Chemoul-Bruyère, André (1938), Compte rendu du concert de J. Laurent-Gallet et Claire Croiza (février 1938), Le Monde musical, $49^{\mathrm{e}}$ année, n ${ }^{\circ}$ 2, p. 48.

[Chevaillier, Lucien] (1929), Compte rendu du concert de la Société musicale indépendante (20 avril 1929), Le Monde musical, 40 année, $\mathrm{n}^{\circ}$ 4, p. 144.

Chevaillier, Lucien (1930), Compte rendu du concert à l'École normale de musique (juin 1930), Le Monde musical, $41^{\mathrm{e}}$ année, $\mathrm{n}^{\circ}$ 6, p. 237.

Cocteau, Jean ([1910]1981), « La patience de Pénélope », dans Théâtre complet, Paris, Gallimard, p. $1419-1460$.

Code, David J. (2012), "A Debussyan Genre. Reflexions on the "Song Triptych" ", L'héritage de Claude Debussy. Du rêve pour les générations futures, colloque international, Montréal, 29 février-3 mars 2012. 
Cools, Eugène (1930), Compte rendu du concert de la Société nationale (3 mai 1930), Le Monde musical, $41^{\mathrm{e}}$ année, $\mathrm{n}^{\circ} 5$, p. 200.

Corbier, Christophe (2010), "La Grèce de Charles Koechlin », dans Philippe Cathé, Sylvie Douche et Michel Duchesneau (dir.), Charles Koechlin, compositeur et humaniste, Paris, Vrin, p. 327-347.

Curzon, Henri de (1932), Compte rendu du concert au Cercle musical de Paris (3 mai 1932), Le Ménestrel, 94 a année, $\mathrm{n}^{\circ} 23$, p. 243, http://gallica.bnf.fr/ark:/12148/bpt6k5621662g/f1.item, consulté le18 janvier 2017.

Dandelot, Georges (1931), Compte rendu du concert de la Société musicale indépendante (28 février 1931), Le Monde musical, $42^{\mathrm{e}}$ année, $\mathrm{n}^{\circ}$ 3, p. 97.

Delage, Edmond (1936), Compte rendu du concert des Concerts Pasdeloup (février 1936), Le Monde musical, $47^{\mathrm{e}}$ année, $\mathrm{n}^{\circ} 2$, p. 48-49.

Delestre, R[obert] (1952), L'ouvre de Marcel Dupré, Paris, Musique sacrée.

Demarquez, Suzanne (1929), Compte rendu du concert de la Société nationale (4 mai 1929), Le Courrier musical, $31^{\mathrm{e}}$ année, $\mathrm{n}^{\circ} 11$, p. 374.

Demarquez, Suzanne (1932), « Euvres de M. Georges Dandelot », Le Courrier musical, 34e année, $\mathrm{n}^{\circ}$ 5,p. 129.

[Demarquez, Suzanne] (1935a), Compte rendu du concert de la Société nationale (9 février 1935), La Revue musicale, $16^{\mathrm{e}}$ année, $\mathrm{n}^{\circ} 3$, p. 208-209.

Demarquez, Suzanne (1935b), Compte rendu du concert de l'Association des jeunes musiciens polonais, Le Courrier musical, $37^{\mathrm{e}}$ année, $\mathrm{n}^{\circ} 4$, p. 84.

De Médicis, François (2010), « Koechlin quartomancien, ou l'art de lire l'avenir de la musique dans les quartes ", dans Philippe Cathé, Sylvie Douche et Michel Duchesneau (dir.), Charles Koechlin, compositeur et humaniste, Paris, Vrin, p. 213-226.

Deré, Jean (1929), Compte rendu du concert de la Société nationale (4 mai 1929), Le Monde musical, $40^{\mathrm{e}}$ année, $\mathrm{n}^{\circ} 6$ p. 227.

Douche, Sylvie (2010), "La mélodie pour voix et piano de 1895 à 1914 ", dans Philippe Cathé, Sylvie Douche et Michel Duchesneau (dir.), Charles Koechlin, compositeur et humaniste, Paris, Vrin, p. 185-209.

Duchesneau, Michel (1997), L'avant-garde musicale à Paris de 1871 à 1939, Sprimont, Mardaga.

Febvre Longeray, Albert (1930), Compte rendu du concert de la Société nationale (3 mai 1930), Le Courrier musical, $32^{\mathrm{e}}$ année, $\mathrm{n}^{\circ}$ 11, p. 342-343.

Flothuis, Marius (1996), "...exprimer l'inexprimable... ». Essai sur la mélodie française depuis Duparc, en dixneuf chapitres et huit digressions, Amsterdam, Rodopi.

Gibbons, William (2008), "Debussy as Storyteller. Narrative Expansion in the Trois Chansons de Bilitis», Current Musicology, $\mathrm{n}^{\circ}$ 85, p. 7-28, http://currentmusicology.columbia.edu/article/debussy-asstoryteller-narrative-expansion-in-the-trois-chansons-de-bilitis, consulté le 18 janvier 2017.

Grayson, David (2001), "Bilitis and Tanagra : Afternoons with Nude Women », dans Jane Fulcher (dir.), Debussy and his World, Princeton, Princeton University Press, p. 117-139.

Hepokoski, James A. (1984), "Formulaic Openings in Debussy ", 19th-Century Music, vol. 8, nº 1, p. $44-59$.

Herstal, Jean d' (1925), Compte rendu du concert aux Amis des lettres françaises (janvier 1925), Le Courrier musical, $27^{\mathrm{e}}$ année, $\mathrm{n}^{\circ} 2$, p. 47.

Himonet, André (1925), Compte rendu du concert de la Société nationale (16 mai 1925), Le Courrier musical, $27^{\mathrm{e}}$ année, $\mathrm{n}^{\circ} 11$, p. 309.

Hoérée, Arthur (1932), "L'Édition musicale. Chant et piano », La Revue musicale, 13e année, n 5 , p. 386-388.

Imbert, Maurice (1929), Compte rendu du concert de la Société musicale indépendante (20 avril 1929), Le Courrier musical, $31^{\mathrm{e}}$ année, $\mathrm{n}^{\circ} 10$, p. 333. 
Imbert, Maurice (1934), «Les concerts symphoniques à Paris », Le Courrier musical, 36 année, nº 1 , p. 16.

Kelly, Barbara (2012), "Writing Early Debussy in Interwar Paris », L'héritage de Claude Debussy. Du rêve pour les générations futures, colloque international, Montréal, 29 février-3 mars 2012.

Koechlin, Charles (1982), "Charles Koechlin (1867-1950). Correspondance », La Revue musicale, $\mathrm{n}^{\text {os }} 348-350$.

Landormy, Paul (1943), La musique française après Debussy, Paris, Gallimard-NRF.

Lapommeraye, Pierre de (1929), Compte rendu du concert de la Société musicale indépendante (20 avril 1929), Le Ménestrel, 91 ${ }^{\mathrm{e}}$ année, $\mathrm{n}^{\mathrm{o}}$ 17, p. 193, http://gallica.bnf.fr/ark:/12148/ bpt6k56173302/f1.item, consulté le 18 janvier 2017.

Launay, Florence (2006), Les compositrices en France au XIX siècle, Paris, Fayard.

Le Coz, Sylvie (1991), Rita Strohl, mémoire de DEA, Université de Rennes 2.

Louÿs, Pierre (1990), Chansons de Bilitis, édition critique par Jean-Paul Goujon, Paris, Gallimard-NRF.

[Mangeot, Auguste] (1935), Compte rendu du concert de l'Association des jeunes musiciens polonais (mars 1935), Le Monde musical, $46^{\mathrm{e}}$ année, $\mathrm{n}^{\circ}$ 3, p. 100.

Niederauer, David J. (1981), Pierre Louÿs. His Life and Art, [Ottawa], Canadian Federation for the Humanities.

Orledge, Robert (1989), Charles Koechlin, 1867-1950. His Life and Works, Chur, Harwood.

Petit, Henri (1933), Compte rendu du concert de Germaine Cernay (mai 1933), Le Courrier musical, $35^{\mathrm{e}}$ année, $\mathrm{n}^{\circ} 11$, p. 282.

Petit, Henri (1935), Compte rendu du concert de Delprat et Cernay (juillet 1935), Le Courrier musical, $37^{\mathrm{e}}$ année, $\mathrm{n}^{\circ} 12-13$, p. 210.

Raugel, Félix (1933), Compte rendu des Concerts Poulet (10 décembre 1933), Le Monde musical, $44^{\mathrm{e}}$ année, $\mathrm{n}^{\circ} 10$, p. 368.

Rumph, Stephen (1994), "Debussy's Trois Chansons de Bilitis. Song, Opera, and the Death of the Subject », Journal of Musicology, vol. 12, n 4, p. 464-490.

Samain, Albert (1924), Euvres, Paris, Mercure de France.

[Samazeuil, Gustave] (1932), Compte rendu du concert à l'École normale de musique (15 février 1932), Le Ménestrel, 94e année, $\mathrm{n}^{\circ}$ 9, p. 96, http://gallica.bnf.fr/ark:/12148/bpt6k5621647s.item, consulté le 18 janvier 2017.

Tolleron, Roger (1935), Compte rendu du concert au Cercle musical de Paris (mai 1935), Le Courrier musical, $37^{\mathrm{e}}$ année, $\mathrm{n}^{\mathrm{o}} 10-11$, p. 182.

Trawel, J. (1931), Compte rendu du concert à la Salle Gaveau (mars 1931), Le Monde musical, 42 année, $\mathrm{n}^{\circ} 3, \mathrm{p} .100$.

Trottier, Danick (2012), « Monumentaliser Debussy. De la grandeur culturelle à la grandeur religieuse », L'héritage de Claude Debussy. Du rêve pour les générations futures, colloque international, Montréal, 29 février-3 mars 2012.

Trottier, Danick (2014), «Creating a Canon. Émile Vuillermoz and French Musical Modernism », dans Barbara Kelly et Christopher Moore (dir.), Musical Criticism in France Interwar Period, Cambridge, Cambridge University Press.

Wenk, Arthur B. (1976), Claude Debussy and the Poets, Berkeley/Los Angeles, University of California Press.

Wheeldon, Marianne (2012), "Debussysme after Debussy ", L'héritage de Claude Debussy. Du rêve pour les générations futures, colloque international, Montréal, 29 février-3 mars 2012.

Youens, Susan (1986), "Music, Verse, and "Prose Poetry". Debussy's Trois Chansons de Bilitis ", Journal of Musicological Research, vol. 7, no 1, p. 69-94. 


\section{Partitions publiées}

Bernard, Jeanne [ca 1933], Trois Chansons de Bilitis, Paris, Senart.

Crocé-Spinelli, Bernard (1904), Pour Bilitis!, Paris, Costallat.

Cuvelier, André-Marie, Chansons de Bilitis, Paris, Jobert. Comprend :

- 1937, recueils 1-5.

$-1939,6^{\mathrm{e}}$ recueil.

-s. d., $7^{\mathrm{e}}$ recueil.

$-1944,8^{\mathrm{e}}$ recueil.

Dandelot, Georges, Quinze Chansons de Bilitis, Paris, Eschig. Comprend :

- 1929, Six Chansons de Bilitis ( $1^{\text {er }}$ recueil).

- 1931, Cinq chansons de Bilitis ( $2^{\mathrm{e}}$ recueil).

- 1933, Quatre chansons de Bilitis (3 ${ }^{\mathrm{e}}$ recueil).

Debussy, Claude (1899), Chansons de Bilitis, Paris, Fromont.

Dupré, Marcel (1920), Deux mélodies tirées des Chansons de Bilitis de Pierre Loü̈s, op. 6, Paris, Leduc.

Fiévet, Paul (1932), Chanson de Bilitis, Paris, Deiss et Crépin.

Grandjean, André [1920], La tristesse de Bilitis. Entr'acte symphonique, Vallauris, Grandjean.

Hasenfeld (1900), "Chanson de Bilitis ", dans Visions brèves. Suite nouvelle d'impromptus et chansons, Paris, Pisa.

Koechlin, Charles ([1908]1923), Cinq Chansons de Bilitis, op. 39, Paris, Senart.

Koechlin, Charles [1909], Mélodies (4e recueil), Paris, Philippo.

Kosma, Joseph (1954), Les chansons de Bilitis. Opérette, Paris, Salabert.

Langlois, Pierre (1904-1905), Chansons de Bilitis, Paris, Dupré.

Le Grand, Robert (1946), Cinq chansons de Bilitis, Paris, Rouart-Lerolle.

Lohné, H. von (1908a), "La quatrième! ». Chanson de Bilitis [...], poésie de Max Rabusson, Paris, Office musical.

Lohné, H. von (1908b), "Le passé qui survit ». Chanson de Bilitis [...], poésie de Max Rabusson, Paris, Matheisen.

Maciejewsky, Roman (2012) [comp. 1932], Pieśni Bilitis, Kraków, Polskie Wydawnictwo Muzyczne.

Mathé, Édouard (1913), Bilitis. Danse grecque, Nice, Decourcelle.

Morales, Gustavo (1930), Deux chansons de Bilitis, Paris, Senart.

Piechowska, Galina (1973), Pieśni Bilitis, Warszawa, Zaiks. Édition française : Chants de Bilitis, Paris, Billaudot, 1977.

Pollet, Marcel (1908), Trois Chansons de Bilitis, Paris, Demets.

Robin, Leo (1920), Chanson de Bilitis, Paris, Morelli.

Strohl, Rita (1900), Bilitis. Poème en 12 chants, Paris,: Toledo.

Thiel, Reynold (1934), Trois chansons de Bilitis, Paris, Hamelle.

Trépard, Émile (1902), "Bilitis! : poèsie d’Arthur Bernède, Paris, Grus.

Wailly, Paul de (1908), "Au vaisseau ", Chanson de Bilitis : deux poèmes antiques, op. 22, Paris, Rouart-Lerolle.

Yzelen, D. (s. d.), Bilitis, Paris, Office d'éditions musicales. 